\title{
A cooperative V2X MAC protocol for vehicular networks
}

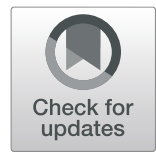

Mohamed A. Abd El-Gawad ${ }^{1,2}$ (D), Mahmoud Elsharief ${ }^{1,3}$ and HyungWon Kim ${ }^{1 *}$

\begin{abstract}
In support of traffic safety applications, vehicular networks should offer a robust Medium Access Control (MAC) layer protocol that can provide a reliable delivery service to safety-related messages. As the safety applications generally use broadcasting to propagate their messages, a reliable broadcast protocol is essential. In general, however, broadcast is considered as unreliable by nature in contrast to unicast. This paper introduces a novel MAC protocol, called a Hybrid Cooperative MAC (HCMAC), which can substantially enhance the reliability of broadcast in vehicular networks by employing a notion of channelization. HCMAC introduces a hybrid protocol that combines a time slot allocation of Time Division Multiple Access (TDMA) and a random-access technique of Carrier Sense Multiple Access (CSMA) and thus minimizes the probability of data collisions. In addition, its feedback strategy further enhances the system performance by preventing transmissions during time slots that experience collisions. Through analysis and simulations, we compare the performance of HCMAC with VeMAC, an existing TDMA protocol. The results demonstrate that HCMAC can offer substantially faster channel access and lower collision rate compared with VeMAC.
\end{abstract}

Keywords: Vehicular ad hoc networks, Reliable broadcast, TDMA, CSMA, Analytical model

\section{Introduction}

In recent years, road safety is receiving increasingly more attention due to the growing number of fatalities and injuries caused by road accidents. Every year, road accidents cause around 1.25 million deaths worldwide [1]. In 2015, 35,092 of 36,973 total transportation fatalities in the USA are caused by highway accidents [2]. The National Highway Traffic Safety Administration (NHTSA) estimates that safety applications provided by vehicle-to-vehicle (V2V) and vehicle-to-infrastructure (V2I) networks could prevent or mitigate $80 \%$ of crashes at intersections and during lane changes. In Dec. 2016, the U.S. Department of Transportation (DOT) announced a new proposed rule that mandates car manufacturers to incorporate $\mathrm{V} 2 \mathrm{~V}$ technologies in all new light-duty vehicles to make the road safer [2]. Consequently, vehicular networks are receiving even higher interest and support from academia, government, and automobile makers than the past years. Vehicular networks can enable us to develop a variety of road safety applications such as lane change warning (LCW), forward collision

\footnotetext{
* Correspondence: hwkim@cbnu.ac.kr

${ }^{1}$ Department of Electronics Engineering, Chungbuk National University,

Cheongju, South Korea

Full list of author information is available at the end of the article
}

warning (FCW), left turn assist (LTA), and intersection management assist (IMA) [3]. In addition, V2V in combination with V2I networks can offer intelligent transportation services, which includes, for example, traffic congestion control and road traffic optimization.

A vehicular network, also known as a vehicular ad hoc network (VANET), is a form of an ad hoc network, in which vehicles communicate with each other. Each vehicle uses an onboard unit (OBU) equipped with one or more radio transceivers and a global position system (GPS). OBUs based on the dedicated short-range communications (DSRC) standard communicate over a $75-\mathrm{MHz}$ spectrum band starting from 5.850 to $5.925 \mathrm{GHz}$. The DSRC standard divides this spectrum to seven channels of $10 \mathrm{MHz}$ : one control channel $(\mathrm{CCH})$ for control information and short safety messages and six service channels (SCHs) for safety- and non-safety-related applications.

DSRC defines the framework of data transmission process between two communication ends and provides a roadmap for interoperable applications. Under this framework, a number of standards are defined by other organizations. For example, IEEE provides a set of standards such as IEEE 802.11p [4] for wireless access, IEEE 1609.3 [5] for networking services, and IEEE 1609.4 [6] 
for multichannel operations. Society of Automotive Engineers (SAE) has released the Message Set Dictionary standard (SAE J2735) [7], which defines a set of messages that represents the core functions for application development.

SAE J2735 defines the Basic Safety Message (BSM), one of the most frequently used message types for DSRC. A BSM is a broadcast message that contains critical information regarding the vehicle's state (e.g., position, speed, and heading). To realize safety applications, vehicles periodically exchange their BSM messages to track neighboring vehicles and take actions to avoid potential collisions. For instance, the IMA application relies on one-hop broadcasting of BSM messages among the vehicles within the same wireless range.

To make the safety applications acquire accurate vehicle and road information and so take timely actions, reliable transmission of BSM messages should be guaranteed with little latency overhead. Consequently, the underlying Medium Access Control (MAC).

The V2V MAC protocol specified in the current standard of IEEE-802.11p, however, does not provide a reliable broadcast service. Since it is derived from the legacy IEEE-802.11 standard, its Carrier Sense Multiple Access/Collision Avoidance (CSMA/CA) protocol offers collision avoidance mechanism only for unicast packets not for broadcast packets. In other words, the Request To Send/Clear To Send (RTS/ CTS) mechanism of CSMA/CA can alleviate the hidden node problems and reduce data collisions for only unicast packets.

Another limitation resides in the Enhanced Distributed Channel Access (EDCA) of IEEE-802.11p, which provides differentiated service for urgent packets. It assigns a smaller contention window $(\mathrm{CW})$ and a shorter interframe spacing (IFS) to higher priority traffic (e.g., critical safety messages). EDCA, however, cannot utilize its variable $\mathrm{CW}$ for broadcast packets, since the current IEEE-802.11p standard cannot detect collisions of broadcast packets. EDCA has another drawback. Although a smaller CW gives a shorter access delay, it increases the probability of collisions, especially when the number of contending vehicles increases [8].

In contrast to the contention-based protocols like IEEE-802.11p standard, other protocols have been proposed to provide more robust wireless access. Time Division Multiple Access (TDMA) or Code Division Multiple Access (CDMA) is among such protocols.

TDMA divides the access time into a group of time slots and allocates each transmitter to the time slots using distributed scheduling schemes. TDMA has been considered by several works [9-17]. RR-ALOHA [9] was originally proposed for mobile networks. It presented a distributed process that allocates time slots, to allow reliable single-hop broadcast. The authors of [10] have proposed an enhancement of RR-ALOHA, called an ADHOC MAC, which employs a slotted/framed structure. In the ADHOC MAC, each node broadcasts the status of all the slots (i.e., the slot is free or busy) in the previous frame. By advertising such information to the neighbor nodes, each node can avoid duplicate reservation of slots and eliminate the hidden node problem. It, however, still has limitations. It suffers from performance degradation for fast-moving nodes, while it does not support the multichannel operation. Thus, it is not compatible with the DSRC standard.

Another TDMA-based MAC protocol, called VeMAC [11], was also proposed for VANET. While it also utilizes the architecture of the ADHOC MAC, VeMAC supports multichannel operation and handles rapid changes in the network topology. In VeMAC, time slots are divided into disjoint sets. In two-direction roads, vehicles moving in each direction are allocated to a different set to reduce data collisions incurred by node mobility. The authors reported that VeMAC achieves higher throughput and assigns time slots much faster than ADHOC. However, it also suffers from critical limitations; access collisions can occur, as two or more vehicles might acquire the same time slot. It can completely fail to detect collisions in certain situations as to be discussed in Section 3.

STDMA [12, 13] is a time-slotted self-organizing MAC protocol which was originally proposed for the vessels industry. STDMA has been in commercial use for Automatic Identification Systems (AIS) [14]. STDMA grants a channel access for all nodes within a bounded delay, regardless of the number of neighbor nodes within the same wireless range. ETSI has evaluated the performance of STDMA by applying it to the cooperative Intelligent Transportation System (C-ITS).

As the vehicular network is highly dynamic in its nature, classical TDMA protocols suffer from frequent rescheduling demand. This limitation motivated many researchers to develop another category of TDMA schemes, called a topology transparent protocol $[18,19]$, which divides road segments into a set of small cells. Each cell is mapped with a group of time slots. Depending on the mapping, each vehicle selects a time slot group based on its location on the road and then acquires a unique time slot from the selected group. The topology transparent protocols, however, have critical restriction that they require a digital map [18].

CDMA is considered in some proposals due to its robustness against noise and interference [20, 21]. It, however, also suffers from its well-known shortcoming: it is very difficult to assign unique pseudo noise (PN) codes to all vehicles, especially in dense networks. If 
different vehicles happen to share the same PN code by distributed PN code selection processes, they can cause highly frequent collisions [21].

This paper presents a novel hybrid MAC protocol, called Hybrid Cooperative MAC (HCMAC), which is based on VeMAC [11] but further optimized for higher performance in vehicular networks. HCMAC takes advantage of the channelization scheme of TDMA and the random-access technique of CSMA. By combining the advantages of TDMA and CSMA strategies, HCMAC can substantially reduce the rate of access collisions in the $\mathrm{CCH}$ channel. Moreover, for the same number of contending vehicles and the available time slots, HCMAC can assign time slots to the vehicles much faster than VeMAC-a key advantage in highly dynamic networks. In addition, HCMAC introduces new techniques for more effective collision detection than conventional protocols. In summary, our contributions are as follows:

- A new MAC protocol that provides a fully distributed time slot reservation and also avoids duplicate time slot allocation

- An analytical model demonstrating that our protocol achieves faster slot reservation

- The proof of the accuracy of the analytical model through simulation results of HCMAC

- Extensive simulation results with highway and urban conditions proving that HCMAC outperforms VeMAC in all metrics: throughput, packet delivery ratio (PDR), inter-packet delay, and the rate of collisions

The remainder of this paper is organized as follows. Section 2 describes the system model, while the details of HCMAC are presented in Section 3. Section 4 analyzes the performance of the HCMAC. In Section 5, simulation results are presented, while the conclusion and future work are given in Section 6.

\section{Methods}

\subsection{System model}

In this paper, we consider the DSRC and IEEE $802.11 \mathrm{p}$ standard for the spectrum and basic link layer protocol, while we propose an enhancement to the link layer. The DSRC standard assigns the channel 178 (i.e., the $\mathrm{CCH}$ ) for control messages and short safety messages (e.g., BSM messages), and other six $\mathrm{SCHs}$ for safety and non-safety applications as shown in Fig. 1 [22]. In our proposed protocol, transmission time over the $\mathrm{CCH}$ or the $\mathrm{SCH}$ is partitioned into frames, while each frame is divided into $S$ equally sized slots. Each slot has two parts: part 1 consists of $W$ equally sized backoff time units (i.e., contention window) used for carrier sensing, while part 2 represents the actual transmission time as illustrated in Fig. 1. While general vehicular networks may include road side units (RSUs), the network topology of this paper consists only of vehicles equipped with an OBU. The OBU includes two radio transceivers and one GPS receiver.

The GPS receiver provides the required synchronization among the vehicles. Hasan et al. [23] show through real experiments that the GPS synchronization accuracy is at tens of nanoseconds which is quite acceptable for the slotted channel concept. We assume that transceiver 1 is tuned to the $\mathrm{CCH}$ to send and receive the periodic BSM messages, while transceiver 2 is tuned to one of the service channels. We assume that all nodes have a fixed wireless range $R$ with the same transmission power level. We also assume that the communication channel is ideal, so transmission failures can be only due to collisions, and the carrier sensing range coincides with the wireless range. Moreover, the channel is assumed to be symmetric. In other words, if node A is in the wireless range of node $B$, node $B$ is also in the wireless range of node $\mathrm{A}$.

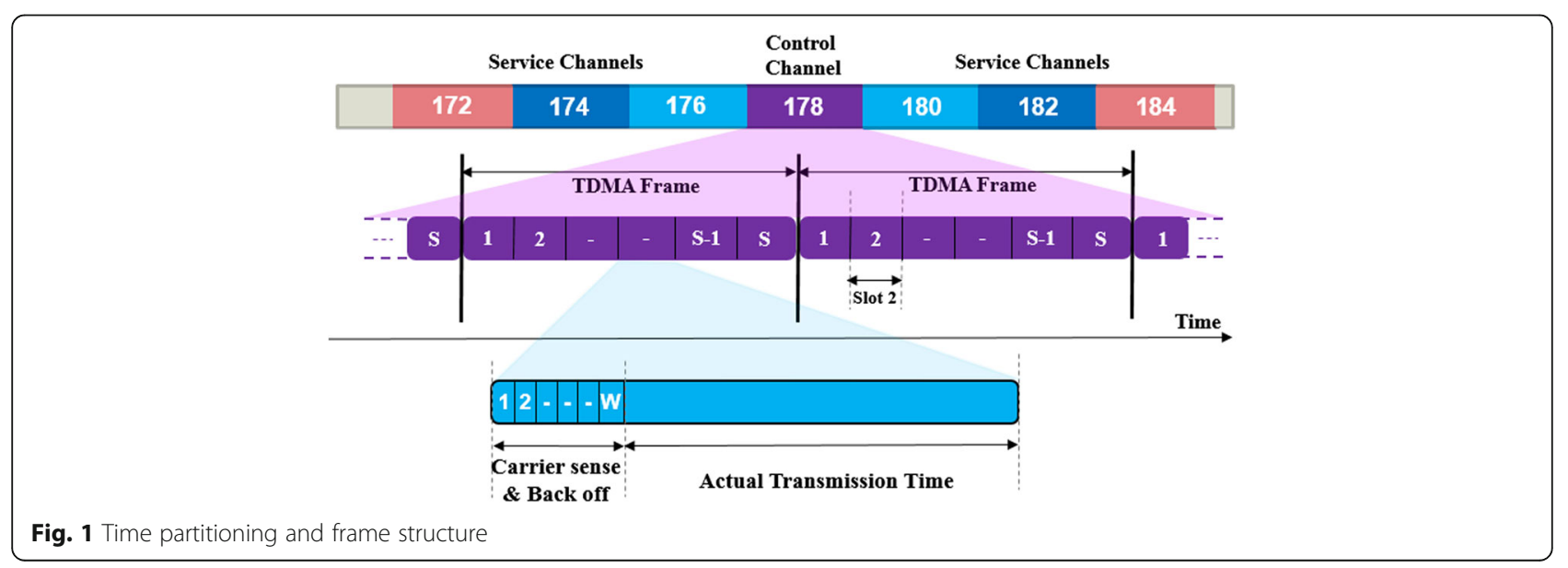


To avoid the problem of hidden nodes, during each vehicle's time slot acquisition process, two sets of neighbors are defined: a one-hop neighbor list, $\mathrm{OH}_{-}$List, and a two-hop neighbor list, TH_List. Each element of the lists consists of the vehicle ID and the acquired time slot of the corresponding vehicle. Each vehicle transmits its updated one-hop neighbor list, as a part of its periodic broadcast messages. Consequently, when the other vehicles receive the one-hop neighbor list, they can determine and update their own two-hop neighbor list. In Fig. 2, for example, vehicle $\mathrm{A}$ has one neighbor in its wireless range, which is vehicle $\mathrm{B}$, and so $\mathrm{OH}_{-} \operatorname{List}(\mathrm{A})$ $=\{(\mathrm{A}, \mathrm{I}),(\mathrm{B}, \mathrm{J})\}$. As vehicle $\mathrm{A}$ can receive vehicle C's one-hop neighbor list via vehicle $B$, vehicle $A$ can add vehicle $C$ in its two-hop neighbor list resulting in $T H_{-} \operatorname{List}(A)=\{(\mathrm{A}, \mathrm{I}), \quad(\mathrm{B}, \mathrm{J}), \quad(\mathrm{C}, \mathrm{K})$. By utilizing such two-hop neighbor lists, each vehicle can substantially alleviate hidden node problems during its time slot acquisition process.

\section{HCMAC protocol}

In this section, we present our proposed MAC protocol, HCMAC. The HCMAC is a novel hybrid MAC protocol that is aimed at improving the performance of the vehicular networks based on the conventional CSMA protocol. It combines the advantages of TDMA and CSMA. It introduces a slot acquisition process that utilizes the CSMA technique in the selected slot. In HCMAC, each node acquires a time slot in a distributed manner. Initially, the vehicle tracks all its neighboring nodes to avoid duplicate slot acquisition. Due to mobility, vehicles that have the same acquired slot might approach each other causing collisions. Even in such events, the vehicles can detect the collisions more effectively, and change their time slot that is a duplicate

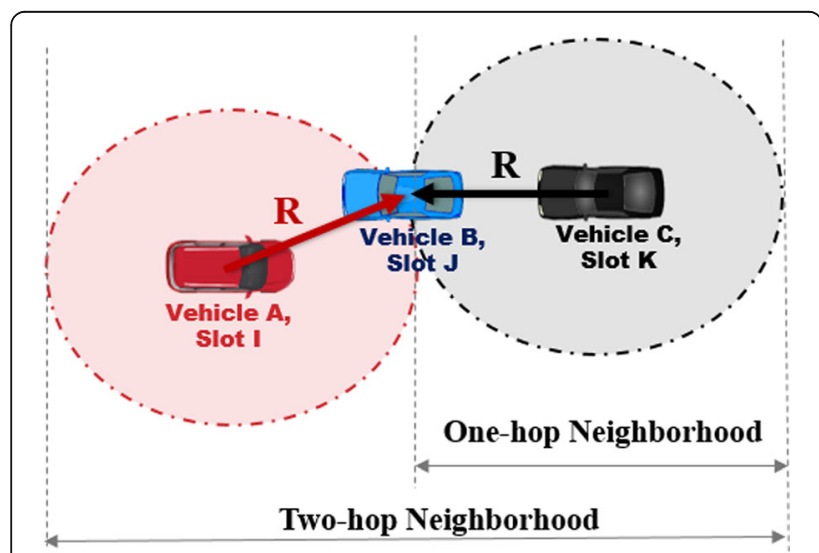

Fig. 2 Two-hop neighborhood concept selection, if the proposed collision detection techniques are employed.

In the following subsections, we describe (a) the packet format and the information exchanged among neighboring nodes, (b) the slot acquisition process of a vehicle joining the network, and (c) the collision detection mechanisms.

While we limit our discussion only to single channel case in this paper, HCMAC can operate with multi-channels, and so it conforms to the DSRC standard.

\subsection{Packet format}

According to IEEE 802.11p, IEEE 1609 family, and SAE J2735 standards, a BSM packet carries the sender vehicle's dynamic status including the position, heading, speed, acceleration, and brake status. As mentioned earlier, in HCMAC, to avoid the slot duplication and the hidden node problem, each vehicle checks the surrounding vehicles during the process of time slot acquisition by inspecting the two-hop neighbor list, $\mathrm{TH}_{-}$List. In HCMAC, each vehicle adds its one-hop neighbor list, $\mathrm{OH}_{-}$List, to the BSM packet's payload. The vehicles exchange the lists among all the neighbors and merge them to construct the TH_List. In order to detect collisions, we defined the slot error (SE) list, which indicates the slots that experience collisions. Figure 3 shows the proposed packet format including the new data fields for $\mathrm{OH}_{-}$ List and SE list.

\subsection{Slot acquisition process}

By employing the packet format shown in Fig. 3, each vehicle acquires a time slot in a distributed way, and so HCMAC does not incur the overhead for expensive centralized decisions. By exchanging $\mathrm{OH}_{-}$List through periodic BSM packets, vehicles can discover the network topology, determine the time slots already occupied, and track any topology change that might occur due to vehicles' mobility.

Let $V_{c}$ be the current vehicle that attempts to acquire a slot. In the start-up phase, $V_{c}$ configures its radio transceiver to the listening mode and then listens to the $\mathrm{CCH}$ channel for $S$ successive time slots. During this listening period, $V_{c}$ receives a one-hop neighbor list, $\mathrm{OH}_{-} \operatorname{List}\left(V_{i}\right)$, from each neighbor $V_{i}$ in its wireless range. By accumulating all $\mathrm{OH}_{-}$Lists, $V_{c}$ determines its own $\mathrm{OH}_{-} \operatorname{List}\left(V_{c}\right)$ and $\mathrm{TH}_{-} \operatorname{List}\left(V_{c}\right)$, a two-hop neighbor list. $V_{c}$ also determines the time slots occupied by the neighbors. Procedure 1 shows a pseudo code that describes the process of the start-up phase in details. 


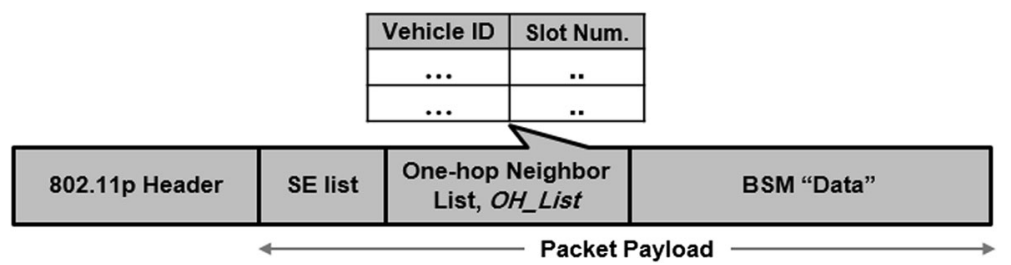

Fig. 3 The packet format

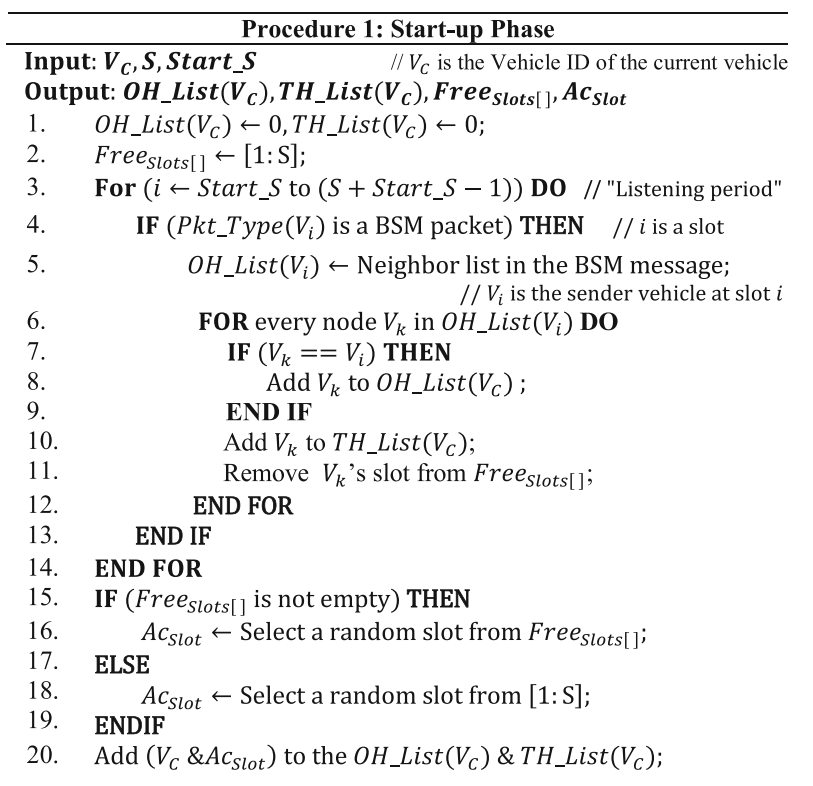

$C$

It takes as inputs $V$, representing the vehicle ID of the current vehicle, $S$ indicating the total number of time slots in one TDMA frame, and Start_ $S$ denoting the slot in which the vehicle joins the network. Vehicle $V$ starts listening to the $\mathrm{CCH}$ and receives its neighbors' packets. Steps 3-14 are conducted, while $V$ receives BSM packets from its neighbors in one full ${ }_{i}$ sequence of slots. Upon receiving a BSM packet from $V$ at slot $i, V$ checks $\mathrm{OH}_{-}$List $(V)$ which is an incremental one-hop neighbor ${ }_{k}$ list of the sender vehicle $V$. If a vehicle ID record, $V$ in $\mathrm{OH}_{-}$List $_{\mathrm{k}} \mathrm{V}$ ) matches the sender's vehicle ID, $V_{C}$ this record $V$ dis stored in both the $\mathrm{OH}_{-}$ $\operatorname{List}(V)$ and $T H_{C} \operatorname{List}(V)$. Otherwise, $V$ is stored only in the $T H_{-} \operatorname{List}(V)$.

Free $_{\text {Slots [ ] }}$ is defined as the set of the available slots. Each vehicle constantly updates its own Free $_{\text {Slots [ ] }}$ by

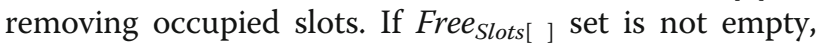
the vehicle randomly picks a free slot from it. Otherwise, it randomly selects any slot from the whole range, $S$, as described in steps 15-19 of Procedure 1. Finally, the vehicle adds $V_{C}$ and the acquired slot, $A c_{\text {Slot }}$, to the neighbor lists as specified by step 20 of Procedure 1 .
Figure 4 illustrates an example of the start-up phase, where vehicle 3 joined two other vehicles (i.e., vehicle 1 and vehicle 2). Starting from the slot that vehicle 3 joined, it listens to the channel for $S$ successive time slots. At slot 4 , vehicle 3 receives a BSM message from vehicle 1 including the $\mathrm{OH}_{-}$List which is the incremental neighbor list of vehicle 1 . Then, vehicle 3 builds its $\mathrm{OH}_{-}$List and $\mathrm{TH} H_{-}$List. As shown in Fig. 4, vehicle 3 inspects its $\mathrm{TH}_{-}$List to determine that slots 1 and 4 are occupied. In this way, during the listening period, vehicle 3 discovers all the surrounding vehicles and their occupied slots, which helps vehicle 3 avoid potential collisions.

\subsection{Collision detection mechanisms}

We first describe how VeMAC detects collisions using its simplistic TDMA protocols, and then introduce HCMAC's enhanced collision detection later. Once the vehicle finished the listening period, it updates the neighbor lists every TDMA frame and transmits at the acquired slot until it detects a collision. To explain the collision detection process, we classify collisions into two cases.

Case 1 is called a "simultaneous-access collision," which occurs when two or more vehicles located within the same wireless range transmit at the same time. This case often occurs when the vehicles join the network at the same time and their randomly acquired slots happen to be the same. Another case is when the vehicles that have the same slot change their locations and move to the same wireless range.

Case 2 is called a "hidden-node collision," which occurs due to the hidden node problem, where a vehicle receives packets from two or more senders located in different wireless ranges. Based on the two collision cases, we define the collision detection mechanisms.

\subsubsection{Detection of simultaneous-access collisions}

We first describe the previous method to identify the collisions of simultaneous-access type followed by HCMAC's enhanced collision detection later. 


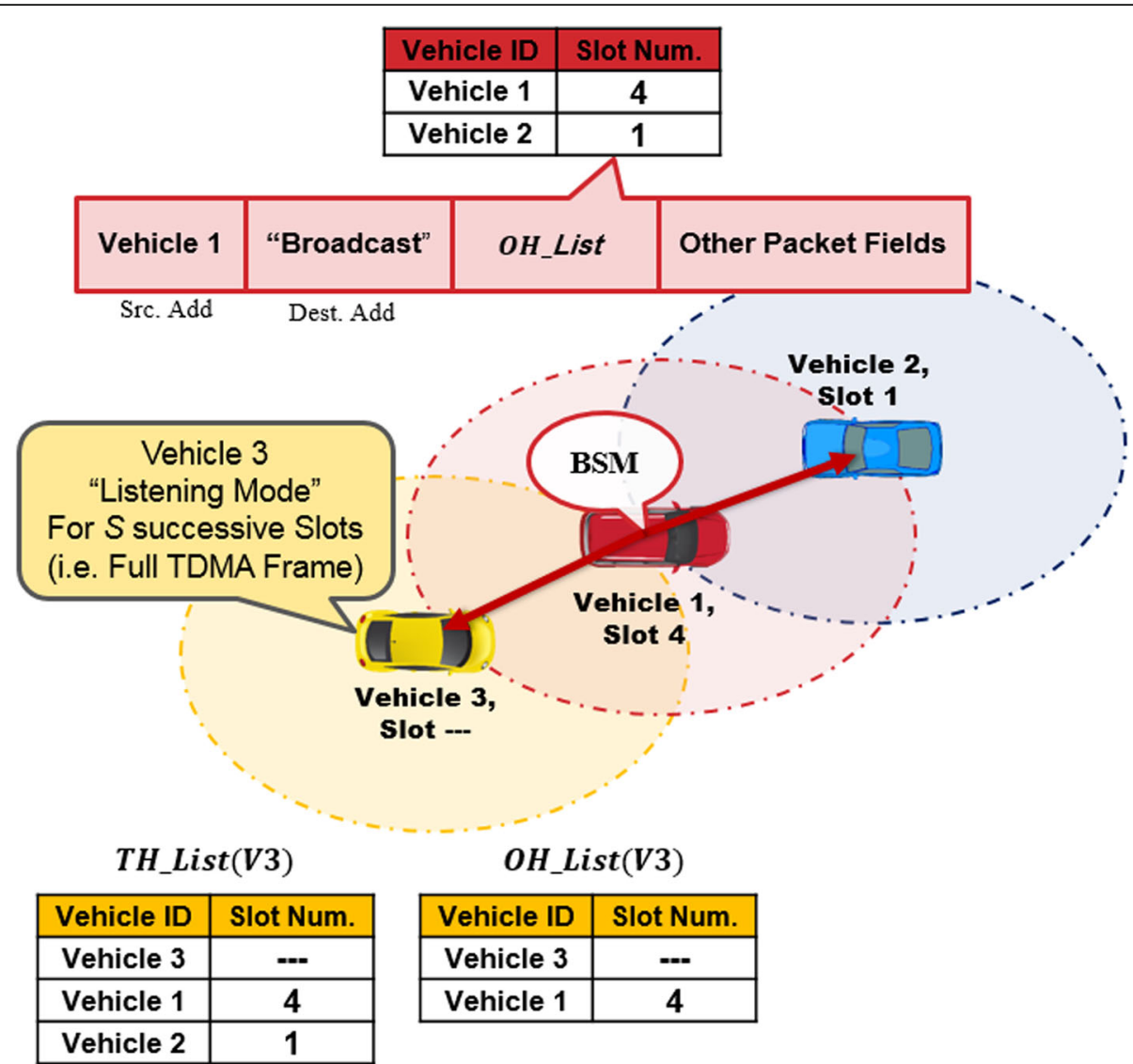

Fig. 4 An example of how each vehicle acquire time slot in the start-up process

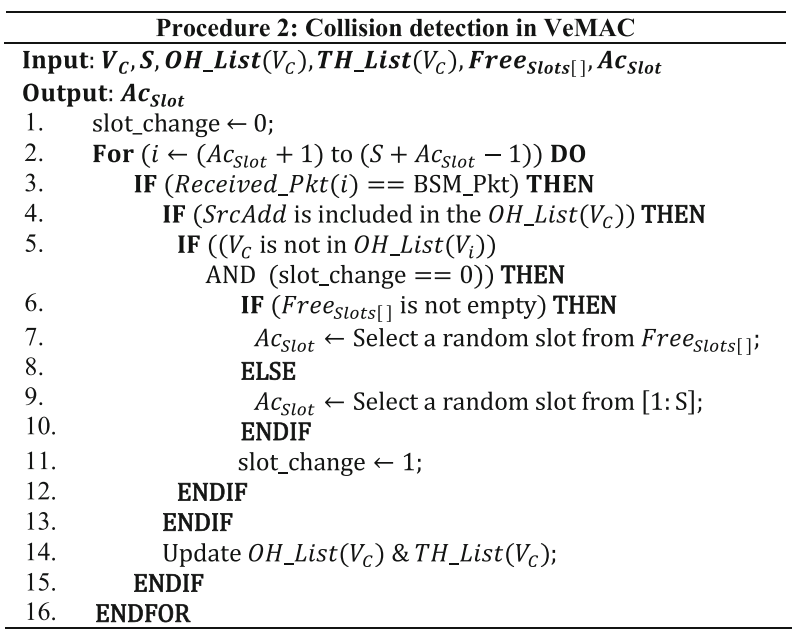

To identify collisions, the previous feedback mechanism $[24,25]$ operates as follows: each vehicle receives a feedback from each neighboring receiver in a form of neighbor list. By inspecting the feedback, each vehicle can determine whether its packet was successfully delivered. By sending $\mathrm{OH}_{-}$List as part of a BSM message, each message can act as an implicit acknowledgment for the previous BSMs received within the same TDMA frame. Figure 5 shows an example of the feedback mechanism for vehicle 1 . According to its $\mathrm{OH}_{-}$List, vehicle 1 expects one BSM message from vehicle 2 at slot 1 and another BSM message from vehicle 3 at slot 10. If these BSMs include vehicle 1 in the $\mathrm{OH}_{-}$List field, vehicle 1 takes them as acknowledgments for the reception of vehicle 1's previous message. If these BSMs do not include vehicle 1 in $\mathrm{OH}_{-}$List, however, vehicle 1 concludes that its previous transmission has failed and selects another slot to avoid successive failures.

VeMAC protocol [11] utilizes the above mechanism for collision detection, where each vehicle needs to wait up to a full TDMA frame (i.e., up to the time it misses one acknowledgment) to check if its previous message is delivered successfully. In Procedure 2, the pseudo code explains how each vehicle detects collisions occurring in its transmitted packets. Steps 3-15 of Procedure 2 check if the received packet comes from a listed neighbor and then verify that the $V_{C}$ (the vehicle ID of the receiver vehicle) is included in the $\mathrm{OH}_{-}$List. If Free Slots[ ] set is not empty, the vehicle randomly picks a new slot from the free slots. 


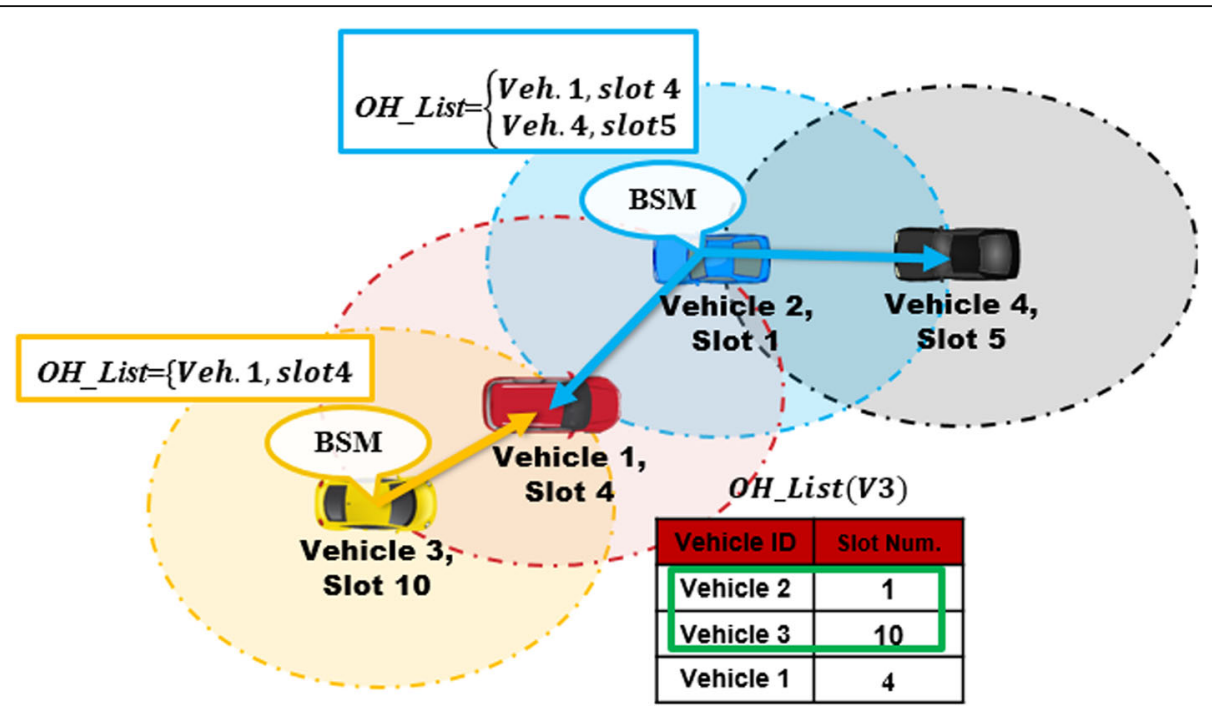

Fig. 5 An example of how BSM messages can act as implicit acknowledgements

Through analysis, we found that although the previous technique provides the benefit of detecting many collisions, it often takes more than one TDMA frame to identify a collision. Furthermore, the previous technique may completely fail to detect collisions in many scenarios. For example, Fig. 6 illustrates a common highway scenario, where vehicle 1 and vehicle 2 both are using the same slot 4 . When vehicle 1 approaches and passes vehicle 2, they transmit at the same time causing a collision. Since they cannot receive any message, they cannot detect the access collision in this scenario. To address this problem, HCMAC provides far more advanced collision avoidance by inserting a contention window of fine grain backoff units in each time slot. Details of HCMAC are described later.

\subsubsection{Detection of hidden-node collisions}

Figure 7 shows a scenario where a hidden node collision occurs. In Fig. 7, vehicle 2 and vehicle 3 enter the wireless range of vehicle 1 in different directions. Suppose that vehicle 1 picked slot 4 , while vehicle 2 and vehicle 3 picked the same slot 10 . If vehicles 2 and 3 entered vehicle 1's wireless range after vehicle 1's time slot (i.e., after slot 4), vehicles 2 and 3 could not receive any information about vehicle 1 . At slot 10 , vehicles 2 and 3 transmit, and a collision occurs at vehicle 1 . Since vehicle 2 and vehicle 3 do not have a neighbor list constructed yet at this moment, they do not expect any acknowledgment from the new neighbor vehicles leaving the collision undetected. In the next frame, at slot 4,

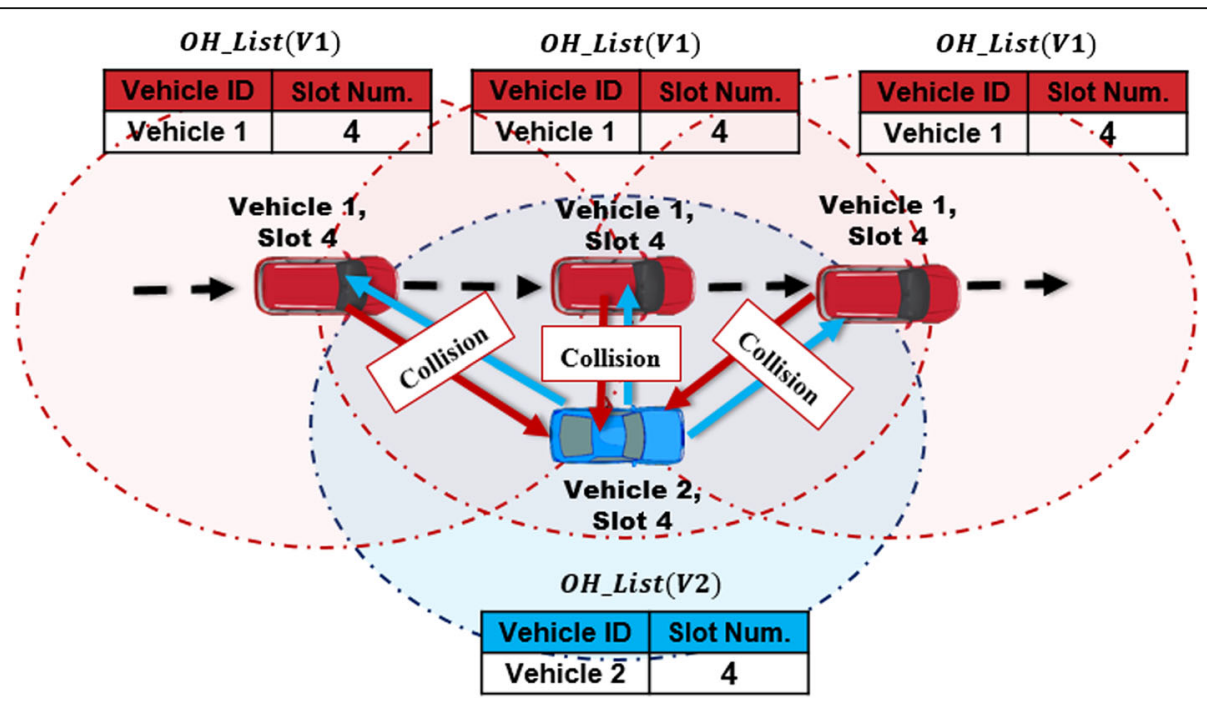

Fig. 6 Detection failure scenario "access collision case" 


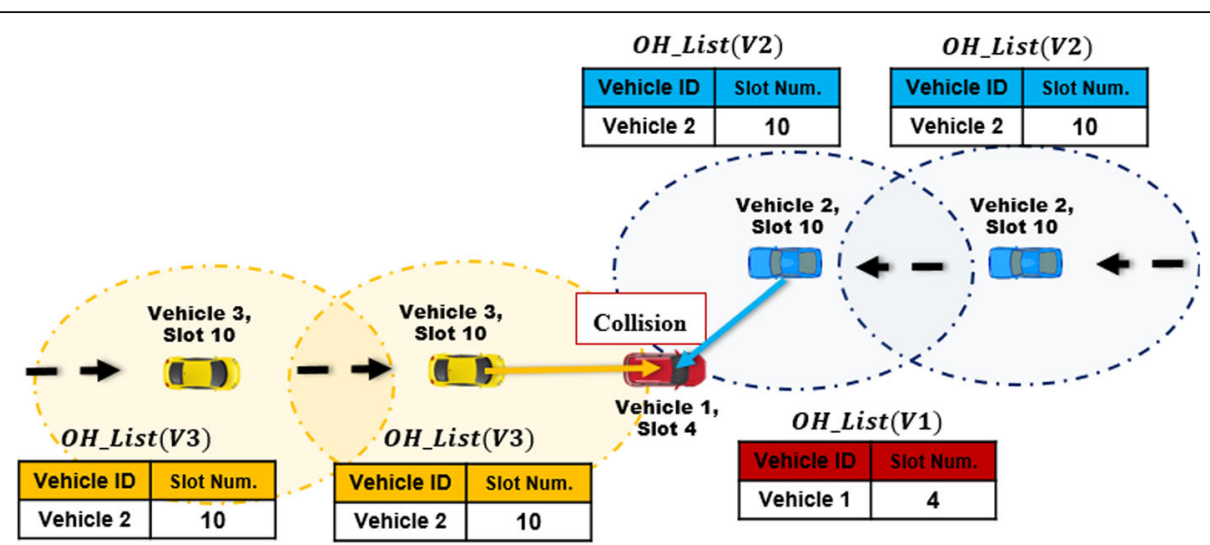

Fig. 7 Long time detection scenario "hidden collision case"

both vehicles 2 and 3 receive a BSM message from vehicle 1 , but they regard vehicle 1 as a new neighbor, and thus they would not expect an acknowledgment for their previous BSM. Therefore, vehicles 2 and 3 once again transmit at slot 10 , and so they waste another frame without knowing that their previous two consecutive BSMs are lost. In the next frame, finally, vehicles 2 and 3 wait for an acknowledgment from vehicle 1 at slot 4 . Vehicle 1, however, transmits without including vehicles 2 and 3 in the $\mathrm{OH}_{-}$List. Vehicles 2 and 3 finally can conclude that their previous packets ran into a collision, and they decide to change their slots.

Although vehicles can detect the collision eventually, it took more than one full TDMA frame. There are various other collision scenarios that can occur very often due to the mobility and the rapid change in the network topology. Therefore, the previous method relying just on the simplistic TDMA with implicit acknowledgement from the receiver nodes cannot ensure highly reliable delivery of broadcast messages like BSMs. This behavior motivates us to add two additional techniques to HCMAC in addition to the implicit acknowledgements: carrier sensing and feedback of slot error list.

\subsubsection{Carrier sensing}

Before transmitting the BSM at the acquired slot, each vehicle waits for a backoff interval, $B$, which is randomly selected out of the contention window, $W$. After this backoff time, the vehicle conducts carrier sensing like in CSMA. If the vehicle detected a carrier or transmitted signal exceeding the threshold of reception signal power, it defers its transmission and selects another time slot. In HCMAC, each vehicle randomly selects a pair $\left(A c_{\text {Slot }}\right.$ $B$ ), where $A c_{\text {Slot }}$ is an available time slot (or random slot if no available slot is left), while $B$ is a random backoff interval. Utilizing these two levels of random selection of channel access time substantially reduces the probability of multiple vehicles' transmitting in the same slot. In addition, it enables faster discovery of the potential collisions.

Figure 8 illustrates an example of HCMAC which employs the carrier sense function at the beginning of each time slot. This example configures the network with five time slots per a TDMA frame, while adding to each slot a contention window of $W=5$. Figure 8 shows four vehicles. Suppose that vehicle 3 and vehicle 4 joined the network almost at the same time, and they selected the same time slot, slot3. Due to their different backoff interval, however, vehicle 4 can transmit safely as it has a lower value, $B=3$. On the other hand, vehicle 3 can detect the transmission of vehicle 4 and select another free time slot.

\subsubsection{Feedback of slot error list}

When the physical layer cannot decode the packet, and the received data produces a Cycling Redundant Check (CRC) error while the received signal strength is higher than the receiver sensitivity, we conclude that there might be a collision. When the received signal strength indicator (RSSI) is above the receiver sensitivity, in most of the real cases, this CRC error happened because of the collision. By employing such a technique, the physical layer can deduce that a collision occurred.

By conveying the RSSI level to the MAC layer, each vehicle can construct a slot error (SE) list consisting of the slots that experienced collisions during the previous TDMA frame. By adding an SE list to the BSM packet, HCMAC allows the receiver to detect various hidden collisions; see Fig. 3 for the packet format. Figure 9 shows the same example illustrated in Fig. 7 and now shows the situation when the first collision happens. Then, vehicle 1 transmits its next packet including the SE list which notifies vehicles 2 and 3 that slot 10 had a 


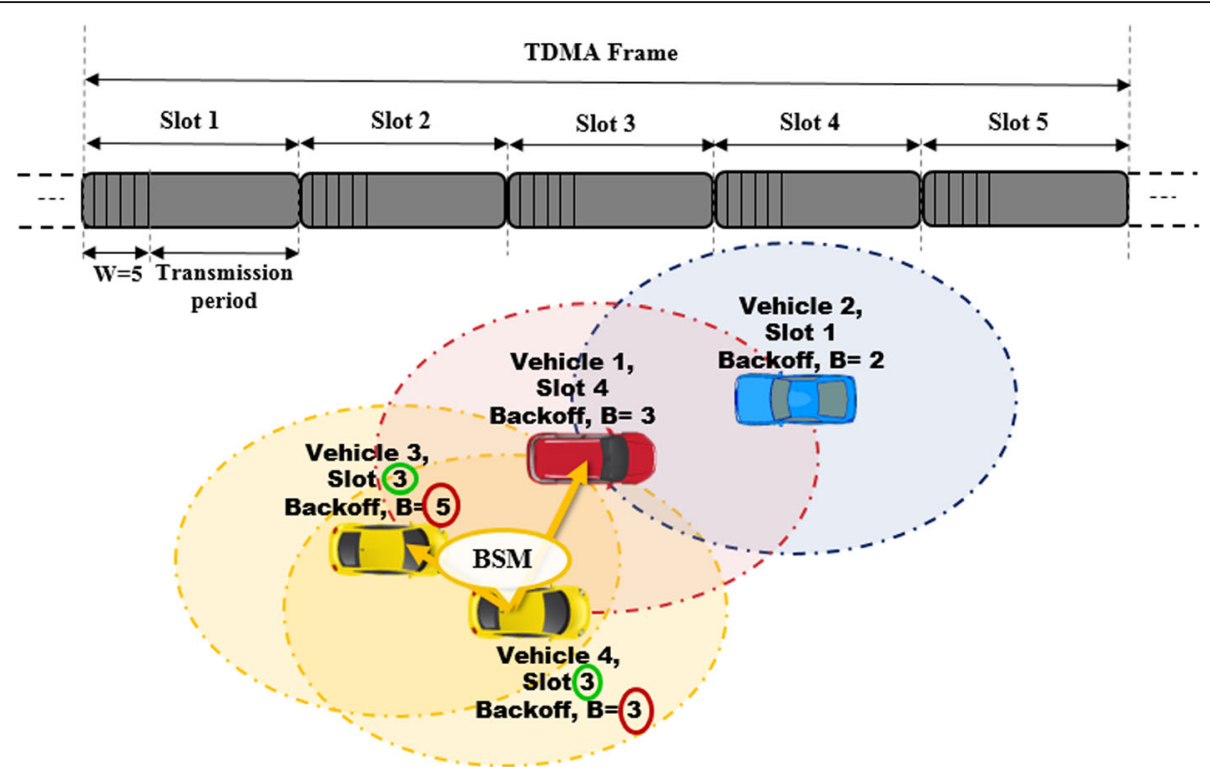

Fig. 8 Mitigating the access collisions by utilizing the carrier sense at the beginning of each time slot

collision in the previous frame. Therefore, vehicles 2 and 3 can avoid successive collisions by acquiring new slots.

The proposed sending and receiving functions of HCMAC including the new collision detection mechanism are illustrated by the pseudo codes in Procedure 3 and Procedure 4. In Procedure 3, at the acquired slot, $A c_{\text {Slot }}$, the sending function observes the channel during the backoff time, $B$. If the channel is clear, it transmits. Otherwise, it selects another free slot. In Procedure 4, steps 4-10 check the SE list. If the SE list includes the acquired slot, $A c_{\text {Slot }}$, of the receiving vehicle, the receiver selects another slot. On the other hand, steps 11-20 utilize the feedback mechanism similar to the one employed by VeMAC's Procedure 2.

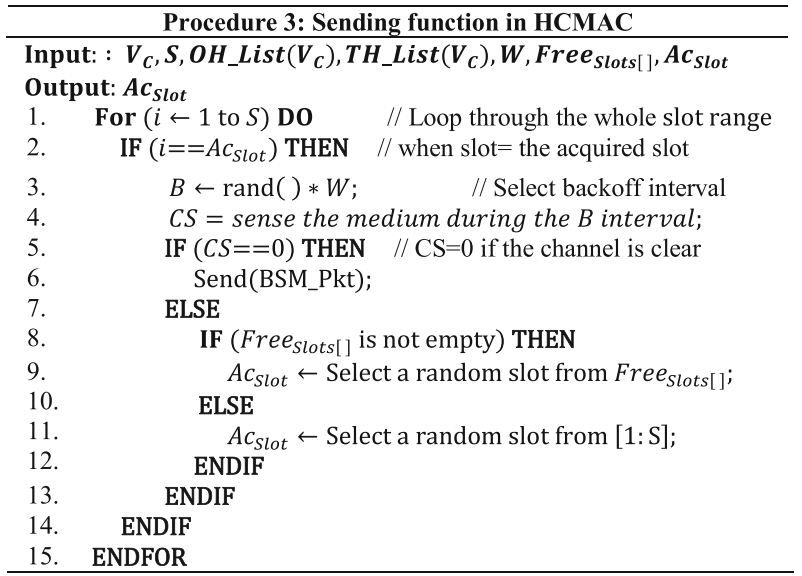

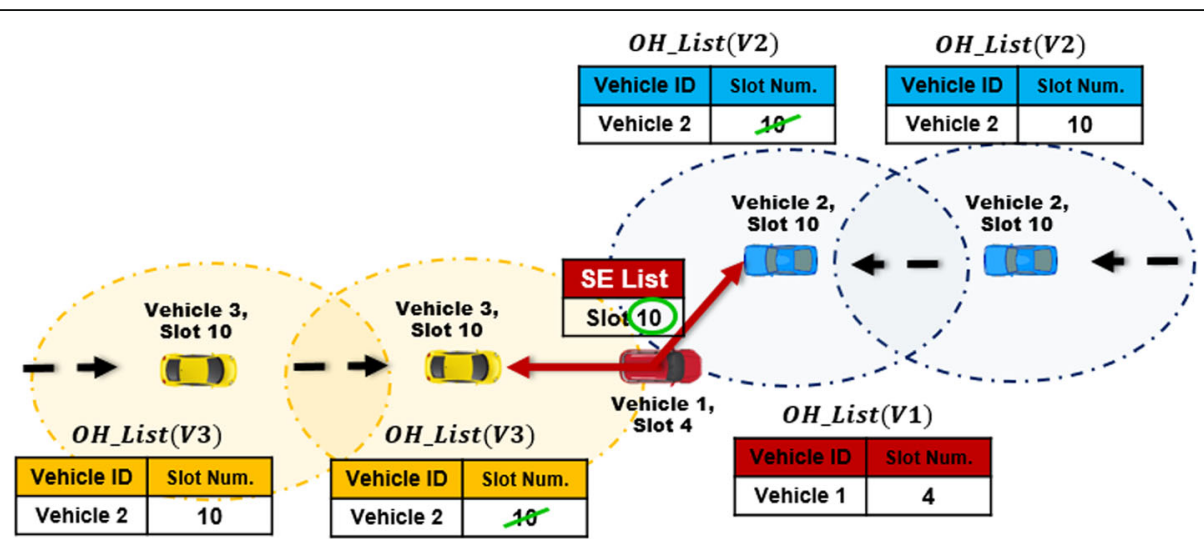

Fig. 9 Fast collision detection by utilizing the SE list 


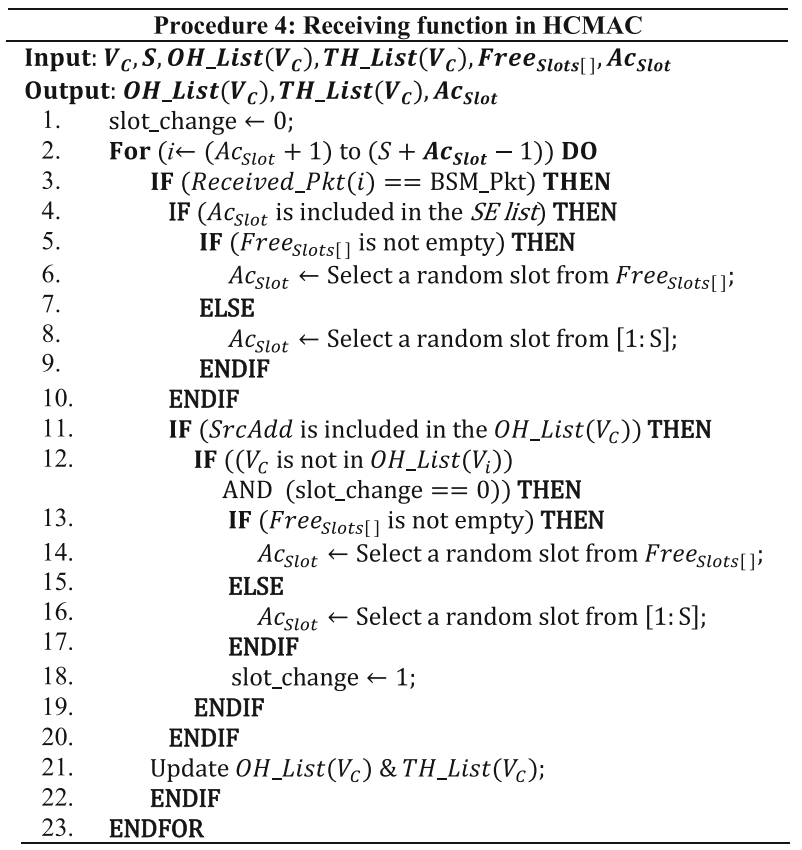

\section{Performance analysis}

In this section, we introduce an analytic model to evaluate the performance of the proposed HCMAC protocol and compare it with VeMAC. We compute the average probability of successful slot acquisition when vehicles join the network. We also express the delay of slot acquisition process, which is the time it takes for all vehicles to successfully acquire unique time slots.

To develop the analytical model, we extend the probabilistic model used in [26] by introducing our parameters for the TDMA slot $A c_{\text {Slot }}$ and backoff interval $B$. To derive mathematically feasible formulation, we follow the assumptions described in [27] with our new parameters additionally considered:

a) $V$ vehicles join the network, each of which attempts to acquire its time slot

b) All the vehicles belong to the same two-hop neighbor list, TH_List (i.e., all vehicles are within the communication range of each other). During the slot acquisition process, we assume that the $T H_{-}$ List does not change for a short instance of time

c) Given a set $S$ of available time slots, each vehicle randomly chooses a time slot, $A c_{S l o t}$, and a backoff interval, $B$

d) At the end of each TDMA frame, all vehicles get informed if their trial to access the channel was successful

e) At the end of each TDMA frame, each vehicle knows the slots occupied by other vehicles during the frame, since the vehicles are within their wireless range. Vehicles that failed to acquire a slot use the updated free slot list, Free Slots [ ] $_{\text {, to choose }}$ another slot in the next frame

While the above assumptions are accurate with respect to the proposed HCMAC protocol, assumptions (d) and (e) intentionally impose unnecessary restrictions for the sake of simplicity of modeling. We discuss how the proposed method can further enhance the latency of collision detection by relieving (d) and (e) later.

In the slot acquisition process, $\frac{1}{s}$ is the probability that a vehicle randomly selects a slot out of $s$ available slots with uniform distribution. Suppose that there are no transmissions during the first $(l-1)$ slots (i.e., these slots are not acquired by any vehicle), and then $k$ out of $v$ vehicles transmit at slot $l$. The probability of the above case, denoted by $P(s, l, v, k)(1 \leq l \leq s$ and $1 \leq k \leq v)$, can be modeled through a Bernoulli process as follows:

$p(s, l, v, k)=\left(\left(1-\frac{1}{s}\right)^{\nu}\right)^{l-1} *\left(\frac{\nu}{k}\right)\left(\frac{1}{s-l+1}\right)^{k}\left(1-\frac{1}{s-l+1}\right)^{\nu-k}$

The formula of Eq. 1 can be approximated to the formula of Eq. 2, which is originally described in [26].

$$
P(s, l, v, k)=\left(1-\frac{l-1}{s}\right)^{v} *\left(\frac{v}{k}\right)\left(\frac{1}{s-l+1}\right)^{k}\left(1-\frac{1}{s-l+1}\right)^{v-k}
$$

In Eq. 2, $\left(1-\frac{l-1}{s}\right)^{v}$ is the probability that out of $v$ vehicles, there is no transmission during the first $(l-1)$ slots, and $\left(\frac{v}{k}\right)\left(\frac{1}{s-l+1}\right)^{k}\left(1-\frac{1}{s-l+1}\right)^{v-k}$ is the probability of having $k$ out of $v$ possible transmissions at slot $l$ where $\frac{1}{s-l+1}$ is the probability of randomly select any slot out of the $(s-l+$ 1) upcoming slots.

While in [26] the authors model the broadcast traffic based on IEEE802.11p for the alternating mode (i.e., switching between $\mathrm{CCH}$ and $\mathrm{SCH}$ ) [6], we reformulate the model to extend it for the structure of HCMAC. Thus, we define $X(s, v)$ as the mean number of successful transmissions during one TDMA frame, whereas [26] defines it as the mean number of successful transmissions during one $\mathrm{CCH}$ interval while taking into account the CSMA contention window size.

$$
\begin{aligned}
X(s, v) & =\sum_{l=1}^{s}\{P(s, l, v, 1)[1+X(s-l, v-1)] \\
& \left.+\sum_{k=2}^{v} P(s, l, v, k) X(s-l, v-k)\right\}
\end{aligned}
$$

In Eq. 3, the term $P(s, l, v, 1)[1+X(s-l, v-1)]$ expresses the success probability when we have just one 
successful transmission (i.e., only one vehicle acquires the $l$ th slot). The term $X(s-l, v-1)$ represents the mean number of successful transmissions in the remaining ( $s$ $-l)$ slots. It makes Eq. 3 a recursive form with the parameter $S$ substituted by $S-l$ indicating that the number of available free slots is reduced by $l$ slots as Eq. 3 proceeds the recursive calculation from the first slot to the last slot to encompass one complete TDMA frame. The term $\sum_{k=2}^{v} P(s, l, v, k) X(s-l, v-k)$ denotes the collision cases when two or more vehicles (i.e., $k \geq 2$ ) acquire the same $l$ th slot.

As described in Section 3, in HCMAC, each vehicle conducts a backoff process before transmitting its packet in the acquired slot to reduce the chances of collisions. When the first vehicle that has the lowest backoff interval transmits, all other vehicles hold their transmissions, and they reselect new slots. To derive an analytic model of the backoff process, we extend the model of Eq. 1 . We derive Eq. 4 from Eq. 1 by introducing new parameters, $w$ and $b$. Here, $w$ denotes the contention window size expressed by a group of backoff units, while $b$ indicates the backoff interval in terms of the number of backoff units. Like in Eq. 1, $k$ is the number of the contending vehicles for a given slot. Equation 5 formulates the total probability $P(w, k, 1)$ of achieving one successful transmission when $k$ vehicles select the same $l$ th slot, and they contend through the contention window $w$. We define $Y(s, v, w)$ as the mean number of successful transmissions by incorporating our proposed backoff process as in Eq. 5.

$$
P(w, b, k, 1)=\left(1-\frac{b-1}{w}\right)^{k} *\left(\frac{k}{1}\right)\left(\frac{1}{w-b+1}\right)\left(1-\frac{1}{w-b+1}\right)^{k-1}
$$

$$
\begin{aligned}
P(w, k, 1) & =\sum_{b=1}^{w} P(w, b, k, 1) \\
Y(s, v, w) & =\sum_{l=1}^{s}\{(s, l, v, 1)[1+Y(s-l, v-1)] \\
& \left.+\sum_{k=2}^{v} P(s, l, v, k)[P(w, k, 1)+Y(s-l, v-k)]\right\}
\end{aligned}
$$

\section{Evaluation of the analytical model}

This section compares the performance of HCMAC with VeMAC by using the analytical model presented in Section 4 . In addition, it proves the accuracy of the analytical model by extensive simulation results. By using Eqs. 2 and 3 , we calculate the expected performance of the
VeMAC protocol. Similarly, by using Eqs. 5 and 6, we estimate the expected performance of the proposed HCMAC protocol. For both VeMAC and HCMAC, we compute the mean number of successfully acquired slots during the TDMA frame. To compute all the possible cases, the recursive models of Eqs. 3 and 6 start from $X(S, V)$ and $Y(S, V, W)$, and then recursively calculate all other cases, respectively. Therefore, we compute the probability of acquiring a slot in each protocol by using the following formulas:

For the VeMAC protocol,

$$
P_{\mathrm{VeMAC}}=\frac{X(S, V)}{V}
$$

For the proposed HCMAC protocol,

$$
P_{\mathrm{HCMAC}}=\frac{Y(S, V, W)}{V}
$$

In Fig. 10, we demonstrate the analytical results for both VeMAC and HCMAC. We compute the probability of successfully acquiring a time slot versus the number of vehicles, $V$, while configuring the TDMA frames with a variable number of slots, $S=[10,20,40]$ slots per frame, and a fixed size contention window, $W=5$. Although the authors of VeMAC used a different analytical model [11, 27], our results obtained by Eq. 3 for VeMAC match the results originally presented in [27]. As shown in Fig. 10, HCMAC outperforms VeMAC regardless of the number of vehicles and the number of slots considered in the evaluation. HCMAC approximately achieves at $S=10$ what can be achieved by VeMAC at $S=20$ (i.e., the green and the blue lines in Fig. 10 are very close). Thanks to the proposed backoff and the carrier sensing processes, it is observed that HCMAC significantly improves the probability of successfully acquiring a time slot.

To verify the accuracy of the analytic model presented in Section 4, we compare the evaluation results of the analytic model with the results of a network simulator that we implemented. As both the slot acquisition and the backoff processes rely on random numbers, we have taken an average result from 50 simulation runs. Figure 11 proves that the results of the analytic model well agree with the simulation results under a wide range of $S$ and $V$ values. Figure 12 demonstrates the comparison results given from the simulations of VeMAC and HCMAC. Here, we configured the TDMA frames of larger sizes $S=[50,100,200]$, while setting the number of vehicles up to 100 vehicles and the contention window to 10 backoff units. Like in the case of 


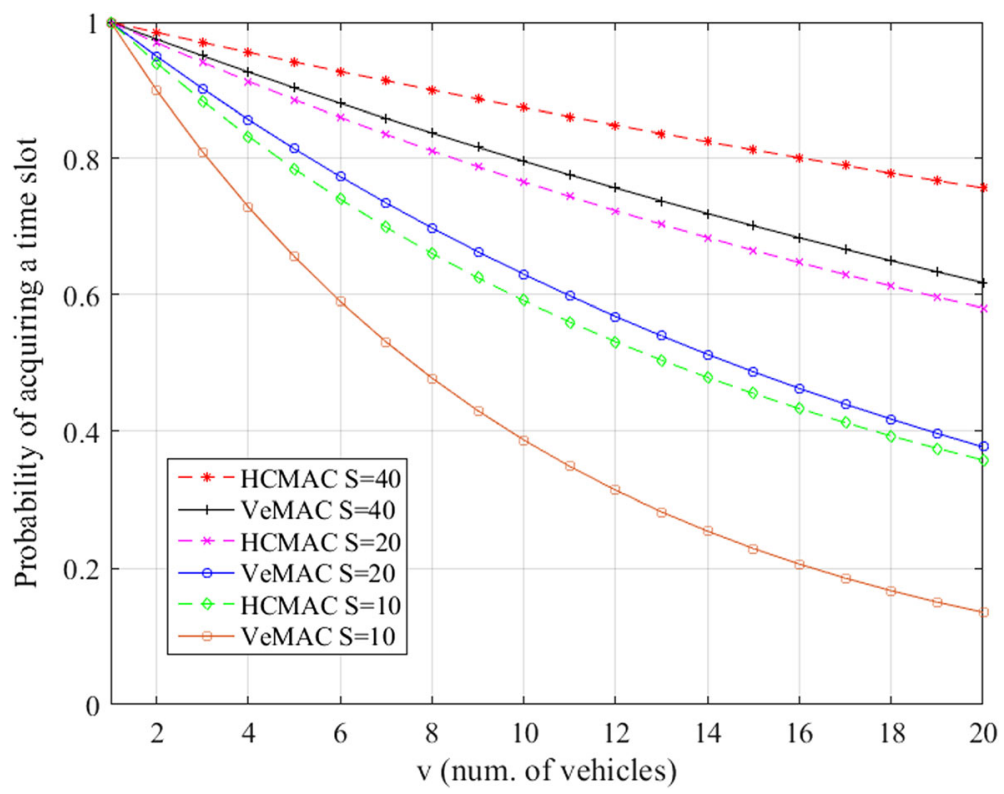

Fig. 10 The probability of acquiring a time slot (analytical results, $S=[10,20,40], W=5$ )

Fig. 10, HCMAC achieves a substantially higher probability of acquiring a time slot compared to VeMAC.

To analyze the impact of the contention window size on the performance of the HCMAC, we used a TDMA frame of a fixed size with a contention window of various sizes. In Fig. 13, we report the analytical results for the probability of acquiring a time slot with $S=10$ and $W=[2,5,10,15,20]$. Figure 13 shows that while the performance increases along with the contention windows of $W=[2,5,10,15]$, the performance levels off with the contention window of size beyond $W=15$.

Similarly, Fig. 14 shows the simulation results of HCMAC obtained with $S=50$ and $W=[2,5,10,20,40]$. It is observed that the probability of time slot acquisition well matches the results of the analytic model of Fig. 13. From Figs. 13 and 14, we can conclude that employing a contention window of a moderate size can provide a considerable improvement in performance, even when the number of vehicles is greater than the number of the

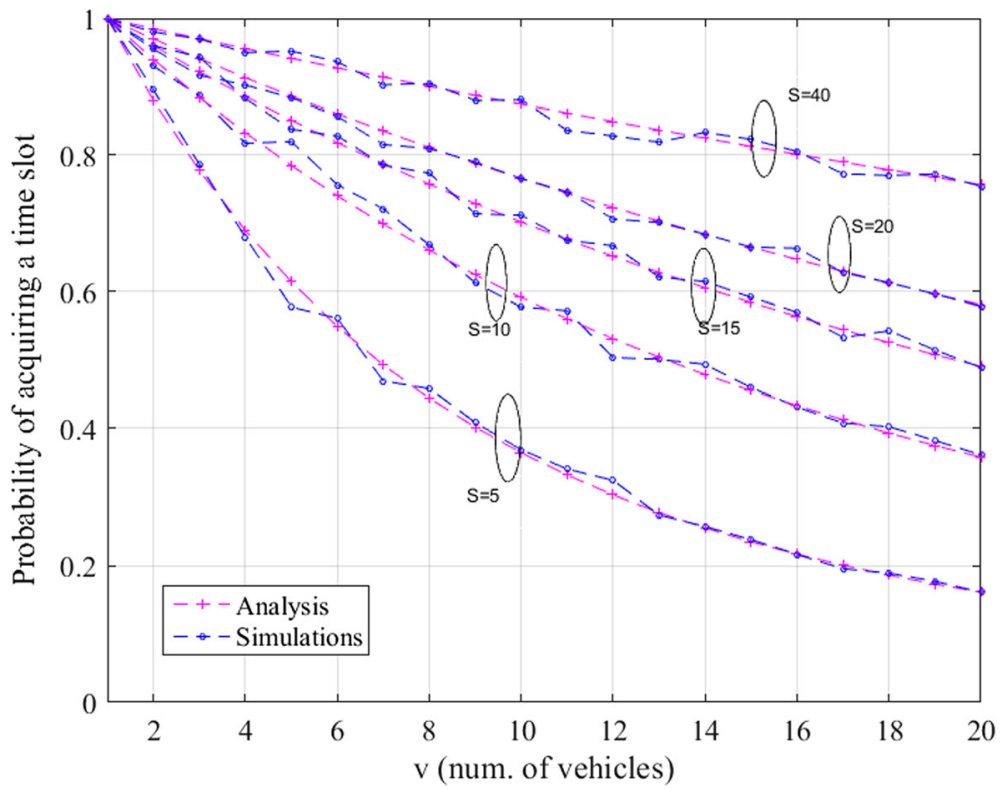

Fig. 11 The probability of acquiring a time slot "HCMAC" (analytical vs. simulation results, $S=[5,10,15,20,40], W=5$ ) 


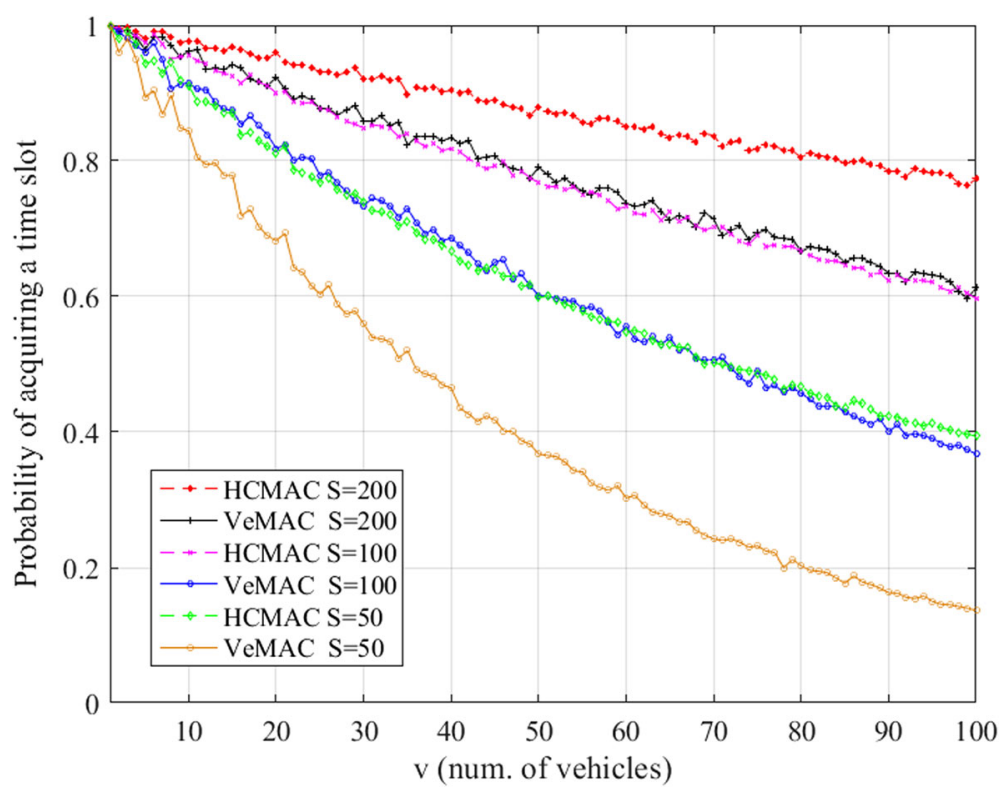

Fig. 12 The probability of acquiring a time slot (simulation results, $S=[50,100,200], W=10$ )

available time slots. Moreover, to express how fast the vehicles can acquire a unique time slot during the slot acquisition process, we evaluate the performance in terms of the required time (i.e., number of TDMA frames) for all vehicles to successfully get a time slot. By utilizing different settings for $S$ and $V$, we demonstrate the average number of vehicles acquiring a unique time slot within $k$ TDMA frames.

In Fig. 15, the analytical results show that vehicles using HCMAC acquire a time slot faster than the vehicles using VeMAC. For example, when $S=15$ and $V$ $=15$, in HCMAC, all vehicles acquired a time slot after five TDMA frames compared to seven frames in VeMAC. To verify the analytical results, we simulated the slot acquisition phase of the HCMAC with different values of $S$ and $V$. We calculated the average number of vehicles which acquire a time slot within $k$ TDMA frames. Figure 16 shows good matching between the analytical and the simulation results. Finally, simulations have been conducted with large values of $S$ and $V$ to

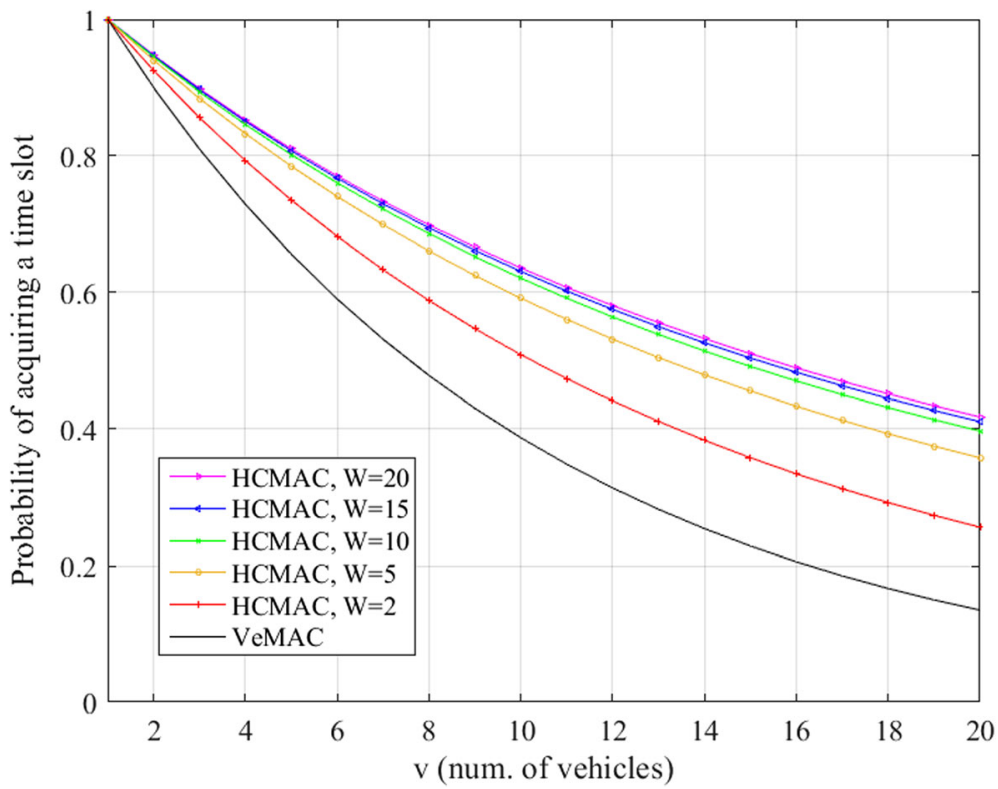

Fig. 13 The probability of acquiring a time slot (analytical results, $S=10, W=[2,5,10,15,20]$ ) 


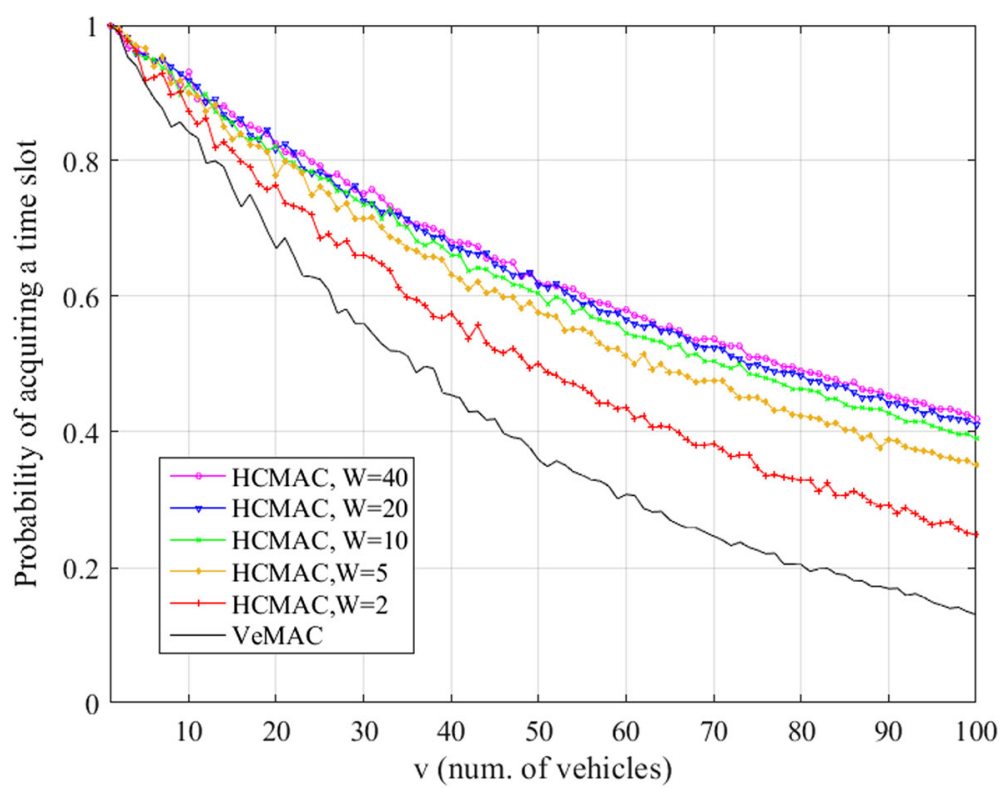

Fig. 14 The probability of acquiring a time slot (simulation results, $S=50, W=[2,5,10,20,40]$ )

show how fast HCMAC acquires the unique time slots compared to VeMAC. Simulation results obtained for VeMAC well match the results originally presented in [27] for all $S$ and $V$ values considered. Figure 17 illustrates the simulation results for HCMAC and VeMAC with different values of $S$ and $V$. It is observed that, for all values of $S$ and $V$, the cases of HCMAC acquire time slots faster than the cases of VeMAC, especially when the number of vehicles, $V$, approaches the number of the available slots, $S$. For example, when $V=90$ and $S=100$, in HCMAC, all vehicles acquire a unique time slot within three TDMA frames compared to five TDMA frames in VeMAC.

\section{Results}

6.1 Implementations and simulations

In this section, we present the network simulator that implements the proposed protocol, HCMAC, and also the previous protocol, VeMAC. We evaluate the performance of HCMAC and VeMAC under various

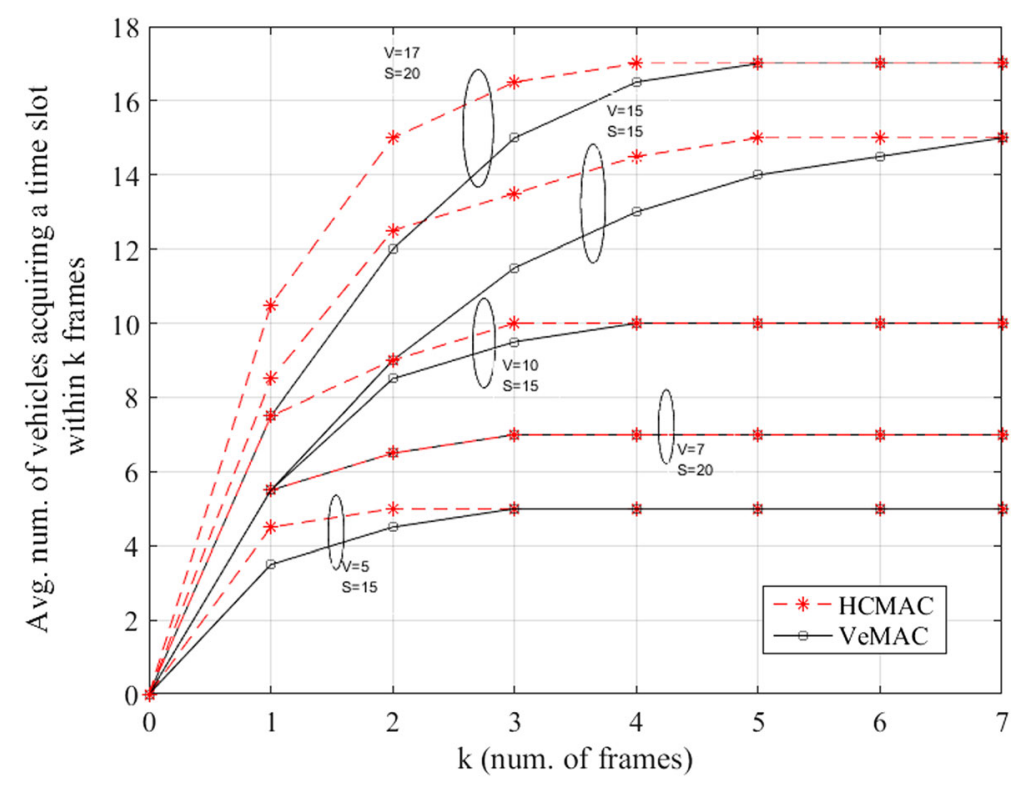

Fig. 15 Avg. number of vehicles acquiring a time slot within $k$ frames (analytical results, $W=5$ ) 


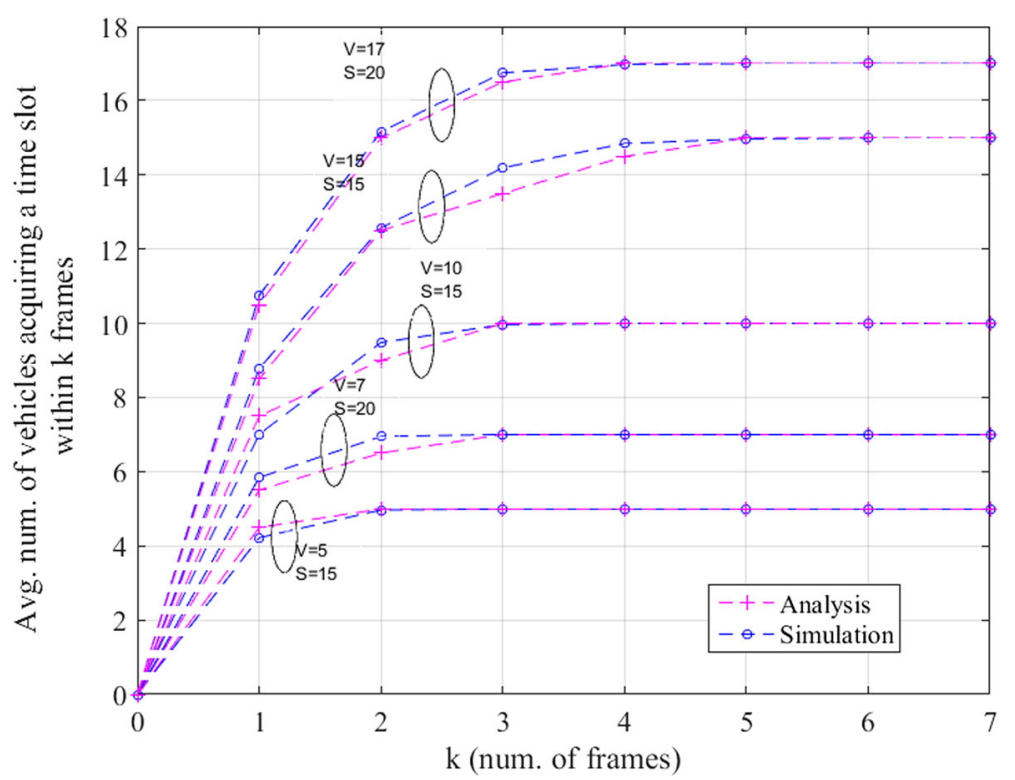

Fig. 16 Avg. number of vehicles acquiring a time slot within $k$ frames (analytical results vs. simulation results, "HCMAC," $W=5$ )

network conditions and demonstrate remarkable performance improvement achieved by HCMAC in comparison with VeMAC.

\subsubsection{Implementation of network simulator}

We implemented the detailed functions of HCMAC in a network simulator using MATLAB environment. While we made restrictive assumptions of (d) and (e) in Section 4 to derive the analytic model with plausible complexity, these restrictions are not needed in the implementation of HCMAC. We therefore implemented the network simulator without the assumptions (d) and (e), which allows early collision detection as follows. In the implementation of HCMAC, when a vehicle experiences a collision at its selected time slot, it can detect the collision within the next slot or in a few slots by analyzing BSMs received from its neighbors. It can figure out whether its previous packet was correctly acknowledged by analyzing a SE list or one-hop neighbor list in the

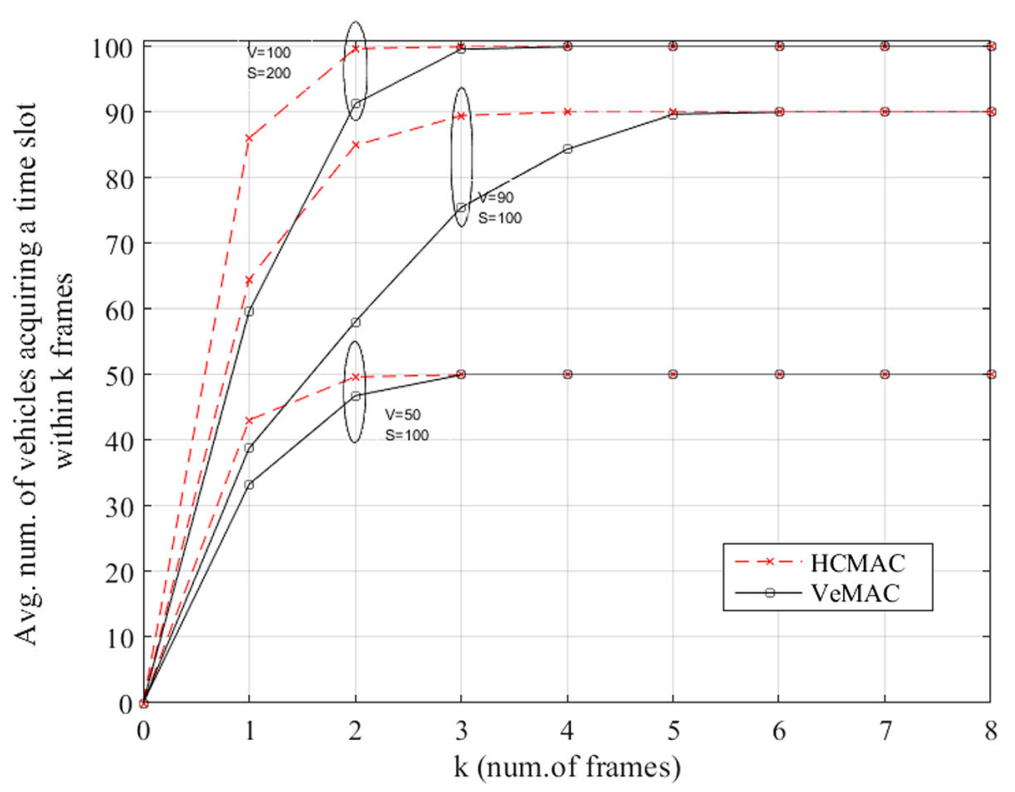

Fig. 17 Avg. number of vehicles acquiring a time slot within $k$ frames (simulation results, $W=10$ ) 
BSMs. Hence, we can reduce the latency compared to the analytic model with the above restrictions.

In our simulator, we considered two propagation models for the wireless channel: ideal and two-ray ground models. For the ideal channel, vehicles can communicate with each other, if they are within a specified wireless range $R$. Therefore, the transmission failure can occur due to the out-of-range condition or transmission collisions. On the other hand, for the two-ray ground propagation model, a successful reception happens only if the received SINR (signal to noise and interference ratio) exceeds a certain threshold. ${ }^{1}$ To conform to the WAVE standards, we configured the periodic safety messages with a report frequency of $10 \mathrm{~Hz}$ which leads to a frame length of $100 \mathrm{~ms}$. We then configured each frame such that it consists of 100 slots, which makes each slot 1-ms long. According to the DSRC standard [7], each vehicle should transmit the periodic safety messages every $100 \mathrm{~ms}$. Therefore, the transmission interval is an important performance metric that can judge whether the MAC protocol satisfies the delay requirement of the standard's safety applications. Each slot is further divided into a 20/80 ratio (i.e., $0.2 \mathrm{~ms}$ for a contention window, while $0.8 \mathrm{~ms}$ for data transmission). The contention window is sub-divided into 10 backoff units leading to each backoff unit of $20 \mu \mathrm{s}$, which is sufficient for HCMAC's carrier sensing process. Figure 14 shows that 10 backoff units represent a reasonable $\mathrm{CW}$ size even in the dense networks tested in this paper. The data rate of all BSM packets in our simulations is set to $12 \mathrm{Mbps}$ with a packet length of 500 bytes, which is larger than a common BSM length. Although we utilize a big size packet compared to the normal size of BSM messages [7], 0.8 $\mathrm{ms}$ is more than enough for transmission according to the analysis made in [3].

In all the simulations presented in this paper, $R$ was set to $150 \mathrm{~m}$. We obtained reliable connections with a distance of $150 \mathrm{~m}$ from the measurement using commercial V2X modules in the actual road conditions. For the two-ray ground channel, vehicles transmit with a TX power of $23 \mathrm{dBm}$ and the successful reception occurs when the received SINR exceeds a threshold of $15 \mathrm{~dB}$. We implemented two network scenarios: a highway scenario of Fig. 18 and an urban scenario of Fig. 19. In both scenarios, we first generate the topology by randomly locating all the vehicles over the road segments. Then, the vehicles start moving and select random time slots to transmit their packets. By receiving the information that is included in the packets (i.e., neighbor list, SE list), each vehicle tries to avoid the collision by avoiding the occupied time slots and re-acquiring free time slots. With the continuous mobility, the topology continuously changes, and each vehicle keeps joining new neighbors and leaving old ones. To test the performance under different vehicle densities, we run the simulation for $2 \mathrm{~min}$ for each generated topology. During the simulation time, we collect all the required data to calculate the performance metrics. In the highway scenario, vehicles move on an eight-lane highway as shown in Fig. 18. Depending on the lanes, each vehicle travels with a constant speed of $60,90,110$, and $120 \mathrm{~km} / \mathrm{h}$ for lanes $1,2,3$, and 4 , respectively. A vehicle can communicate only with the vehicles located within a distance $d \leq R$. To keep the same number of vehicles during the simulation time, a vehicle which reaches one end of the road re-enters from the other end. Also, this keeps changing the topology as the vehicles approaching each other from opposite directions makes each section of the road always have new arrivals. Hence, a continuous time slot rescheduling is always required.

In the urban scenario, there are three vertical and three horizontal bi-directional road segments. In between road segments, there are four building squared blocks. Each vehicle selects a random speed from the interval $40-60 \mathrm{~km} / \mathrm{h}$; then, it continues moving with the same speed during the simulation time. When a vehicle reaches to one of the intersections, it randomly turns to one of the allowable moving directions. In this way,

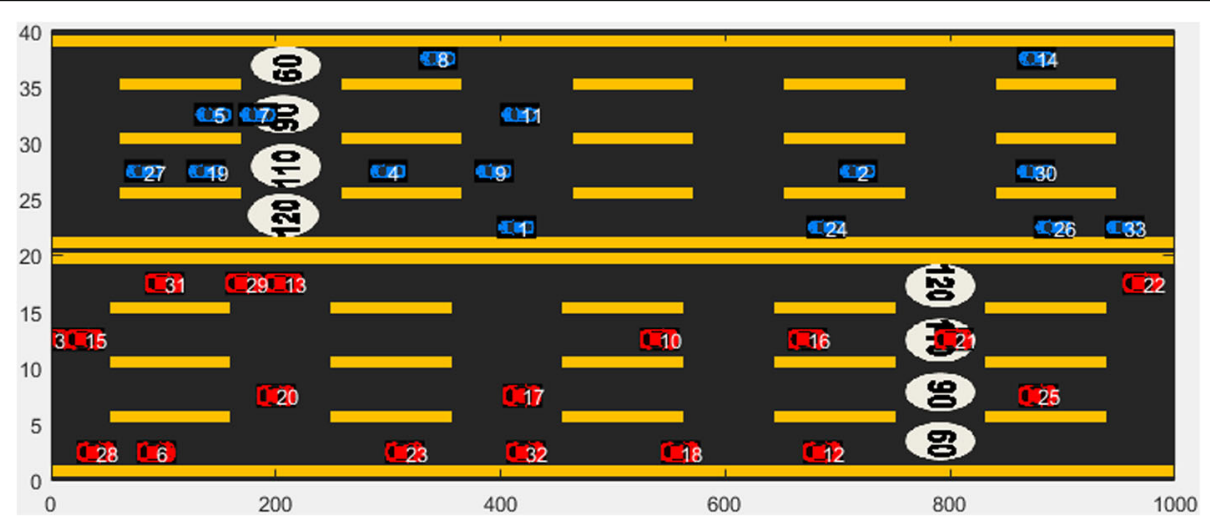

Fig. 18 Highway scenario 


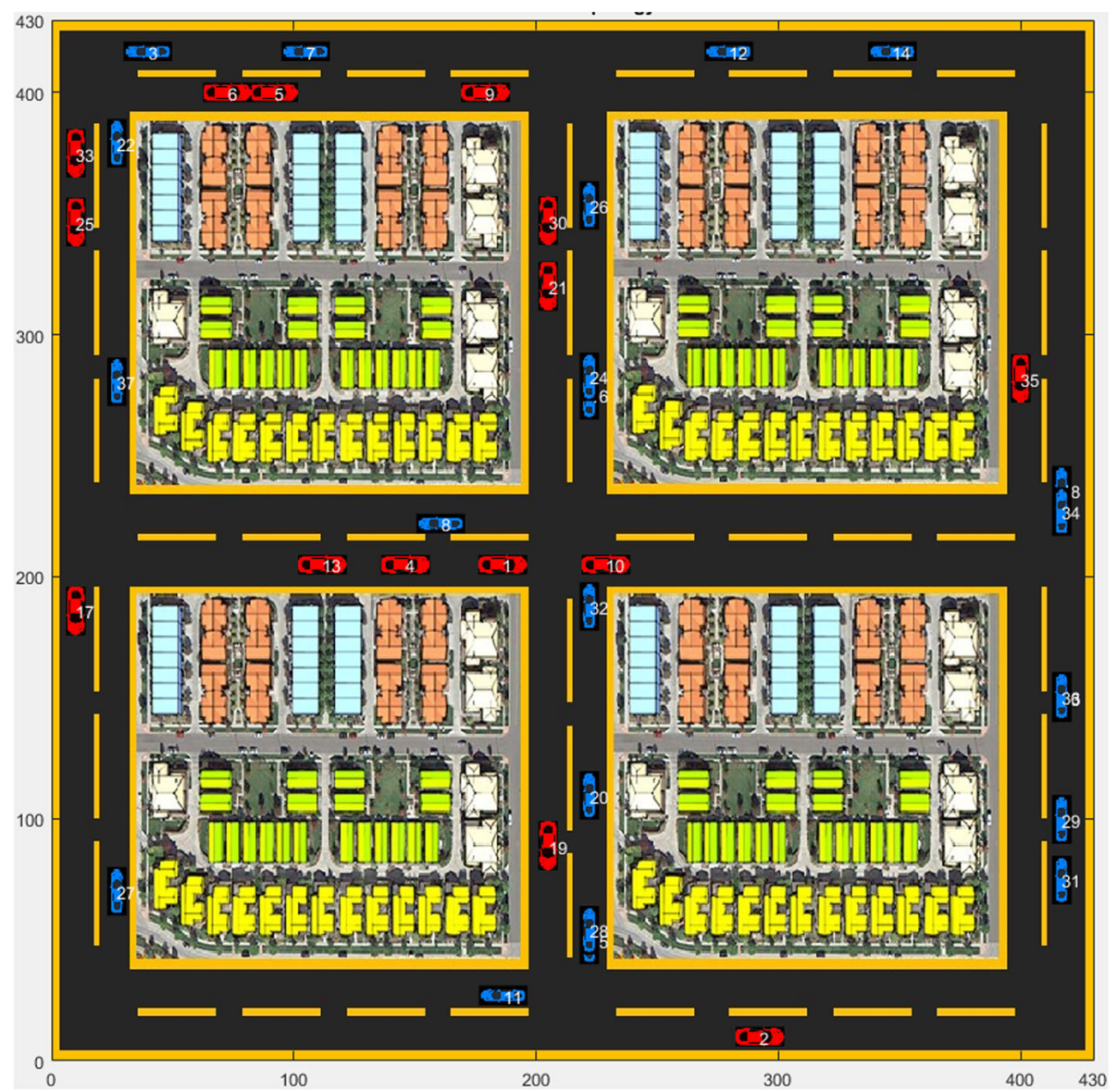

Fig. 19 Urban scenario

vehicles keep moving around, and the number of vehicles remains constant during the simulation period. We also assume that the squared building blocks can totally block the wireless signal. Therefore, away from the intersection zones, a vehicle can only communicate with the vehicles which belong to the same road segment and located at a distance $d \leq R$.

For the simulations, we chose the target network configuration such that the probability of successful slot acquisition is at least $80 \%$ with 100 slots per TDMA frame. We observed that with such network configurations the performance of the networks is acceptable for our target applications.

In Fig. 12 in Section 5, the analytic model demonstrated that the probability of successful slot acquisition becomes greater than $80 \%$ when we have no more than 45 vehicles per wireless range. For instance, in the highway scenario, $1-\mathrm{km}$ road segment can have around seven adjacent wireless ranges, if each wireless range contains 45 vehicles on average. This leads to around $7 \times 45=315$ vehicles $/ \mathrm{km}$. From this perspective, we measured the performance of the considered protocols under different vehicle densities, starting from 50 vehicles $/ \mathrm{km}$ up to 400 vehicles $/ \mathrm{km}$ (i.e., 400 vehicles represent around $120 \%$ of a network load). In this way, the performance can be analyzed in sparse and dense networks including the target network condition. In the same context, we tested the performance for the urban scenario starting with 50 vehicles up to 650 vehicles (i.e., with 650 vehicles, the network load is around $90 \%$ ).

Table 1 summarizes the simulation parameters for the two scenarios such as the road dimension, the properties of packets and time slots, and the number of vehicles in the network. The initial positions of vehicles are uniformly distributed over the simulated road segment of $1000 \mathrm{~m}$ for the highway scenario and $430 \mathrm{~m}$ for the urban scenario, respectively.

\subsubsection{Performance metrics}

We evaluated the performance of various network examples using the simulator described above. We used the following performance metrics to compare the performance between HCMAC and VeMAC: 
Table 1 Simulation parameters

\begin{tabular}{|c|c|c|}
\hline Parameter & Highway & Urban \\
\hline Road segment length & $1 \mathrm{~km}$ & $430 \mathrm{~m}$ \\
\hline Num. of horizontal segments & - & 3 \\
\hline Num. of vertical segments & - & 3 \\
\hline Lane width & $5 \mathrm{~m}$ & $5 \mathrm{~m}$ \\
\hline Num. of lanes per direction & 4 & 1 \\
\hline Wireless comm. range & $150 \mathrm{~m}$ & $150 \mathrm{~m}$ \\
\hline Vehicle speed & {$[60,90,110,120] \mathrm{km} / \mathrm{h}$} & {$[40-60] \mathrm{km} / \mathrm{h}$} \\
\hline Packet size & 500 bytes & 500 bytes \\
\hline Data rate & $12 \mathrm{Mbps}$ & $12 \mathrm{Mbps}$ \\
\hline TX power & $23 \mathrm{dBm}$ & $23 \mathrm{dBm}$ \\
\hline SNR threshold & $15 \mathrm{~dB}$ & $15 \mathrm{~dB}$ \\
\hline Broadcast rate & $10 \mathrm{MHz}$ & $10 \mathrm{MHz}$ \\
\hline Slots per frame & 100 & 100 \\
\hline Slot width & $1 \mathrm{~ms}$ & $1 \mathrm{~ms}$ \\
\hline Contention window size & $0.2 \mathrm{~ms}$ (10 backoff units) & $0.2 \mathrm{~ms}$ (10 backoff units) \\
\hline Number of vehicles & $50-400$ & $50-650$ \\
\hline Number of RSUs & 0 & 0 \\
\hline Simulation time & $2 \mathrm{~min}$ & $2 \mathrm{~min}$ \\
\hline
\end{tabular}

The number of collision events represents the average number of collision events per TDMA frame (two or more collided packets at the same slot are counted as one collision event). This metric considers the access collision and the hidden collision cases as defined earlier. To accommodate hidden collisions, this metric is calculated by considering a two-hop neighborhood.

Network throughput is defined as the average number of the successful transmissions per frame in the one-hop neighborhood. As the MAC protocol becomes more robust with respect to the collisions, the network throughput increases.

Packet delivery ratio (PDR) is defined as the ratio of the number of successful packet receptions to the total number of expected receivers located in the wireless range. PDR often serves as an important metric for the reliability of MAC protocols in general.

Average transmission interval is defined as the average time interval between two consecutive packet transmissions from the same vehicle, which is calculated over all vehicles at all time slots.

Maximum transmission interval is defined as the maximum value of the transmission intervals calculated for each vehicle at each selected slot.

\subsection{Evaluation of simulation results}

In this subsection, we present the simulation results using an extensive set of example networks under the highway and urban scenarios. ${ }^{2}$

\subsubsection{Simulation results under highway scenarios}

Figure 20 shows the number of collision events measured with the increasing number of vehicles. In the sparse networks with the number of available slots larger than the number of vehicles, both VeMAC and HCMAC exhibit very few collisions. When the network becomes denser with 250 or more vehicles, however, the number of collisions starts sharply increasing. HCMAC incurs significantly fewer collisions than VeMAC does in the dense networks. For instance, over the ideal channel, when the number of vehicles is 400, HCMAC experiences only two collisions per frame, while VeMAC has five collisions per frame. The same observation is shown for the two-ray ground channel; HCMAC experiences fewer collisions compared to VeMAC. The good performance of HCMAC is attributed to the proposed collision detection mechanisms based on CSMA and SE list.

Figure 20 also shows that with the two-ray model, each protocol has fewer collision events compared to the ideal model. That is, in the ideal channel case, receiving multiple packets from different transmitters at the same time is considered a collision event, but, on the other hand, with the employed two-ray ground model, the receiver node can still successfully capture one of the transmitted packets if the received SINR exceeds the threshold.

HCMAC's lower collision rate leads to better performance in other metrics as well.

As shown in Fig. 21, HCMAC outperforms VeMAC in the performance of network throughput. For both 


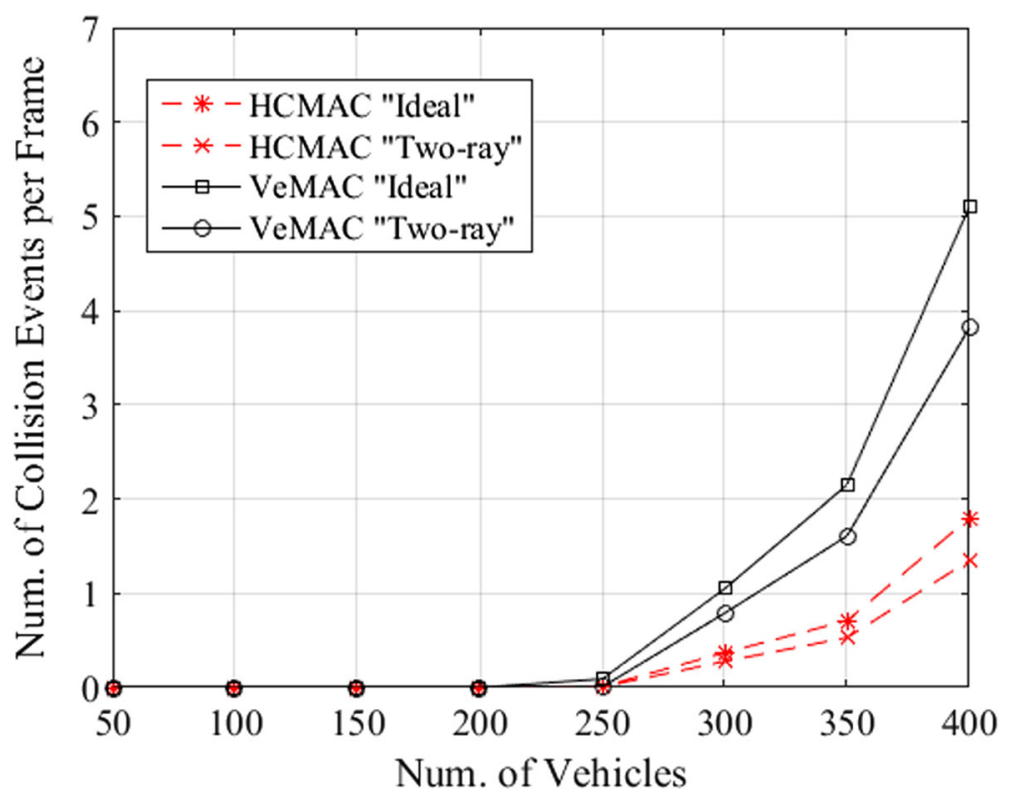

Fig. 20 Number of collision events "highway scenario"

channel models, when the number of vehicles is over 250, HCMAC experiences higher throughput compared to VeMAC. Figure 21 also illustrates that as the two-ray channel model introduces propagation losses, both protocols achieve less throughput compared to the ideal channel case. Figure 22 illustrates average PDR achieved by the two protocols. For the low densities of 250 or fewer vehicles in the network, both HCMAC and VeMAC provide very high PDR (e.g., when the number of vehicles is 150 , PDR is around 99\%).
As the number of vehicles increases, the PDR for VeMAC decreases. In contrast, HCMAC can achieve higher PDR even with higher vehicle densities (e.g., Over the ideal channel, when the number of vehicles is 400 , HCMAC's PDR is around $96 \%$ compared to $87 \%$ for VeMAC. Also, with the two-ray ground model, HCMAC achieves a PDR of $81 \%$ compared to $74 \%$ for VeMAC.).

Finally, we measured the average transmission interval under different vehicle densities as shown in Fig. 23. HCMAC achieves lower interval in the dense networks.

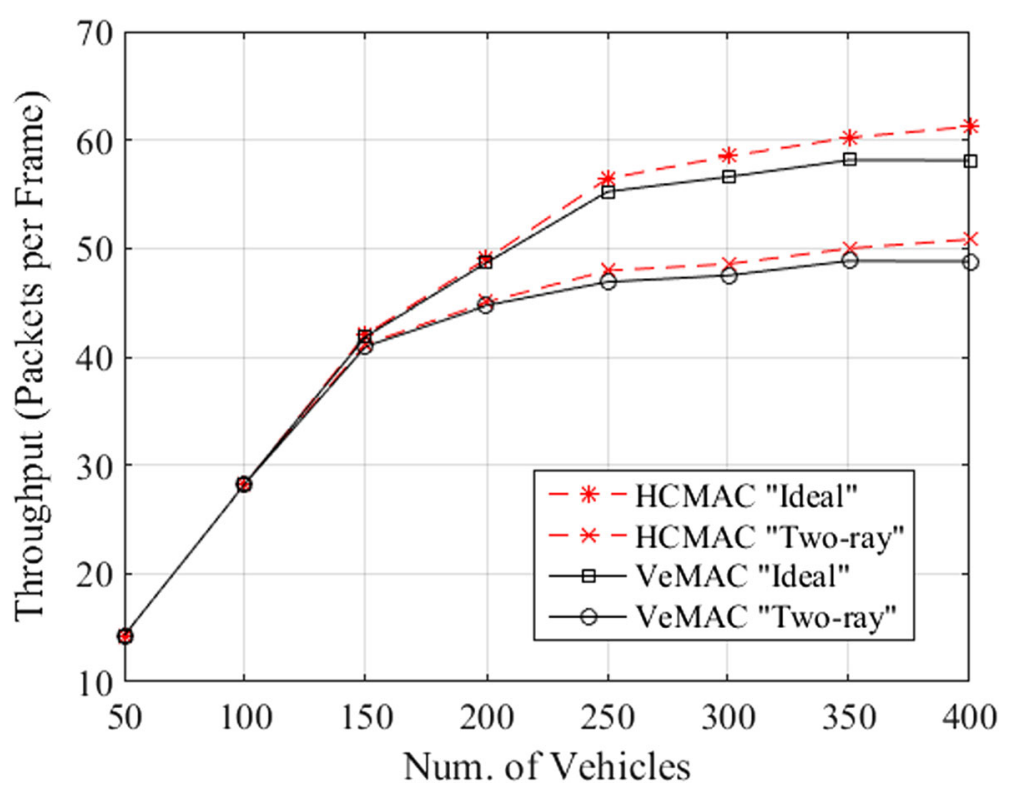

Fig. 21 Throughput "highway scenario" 


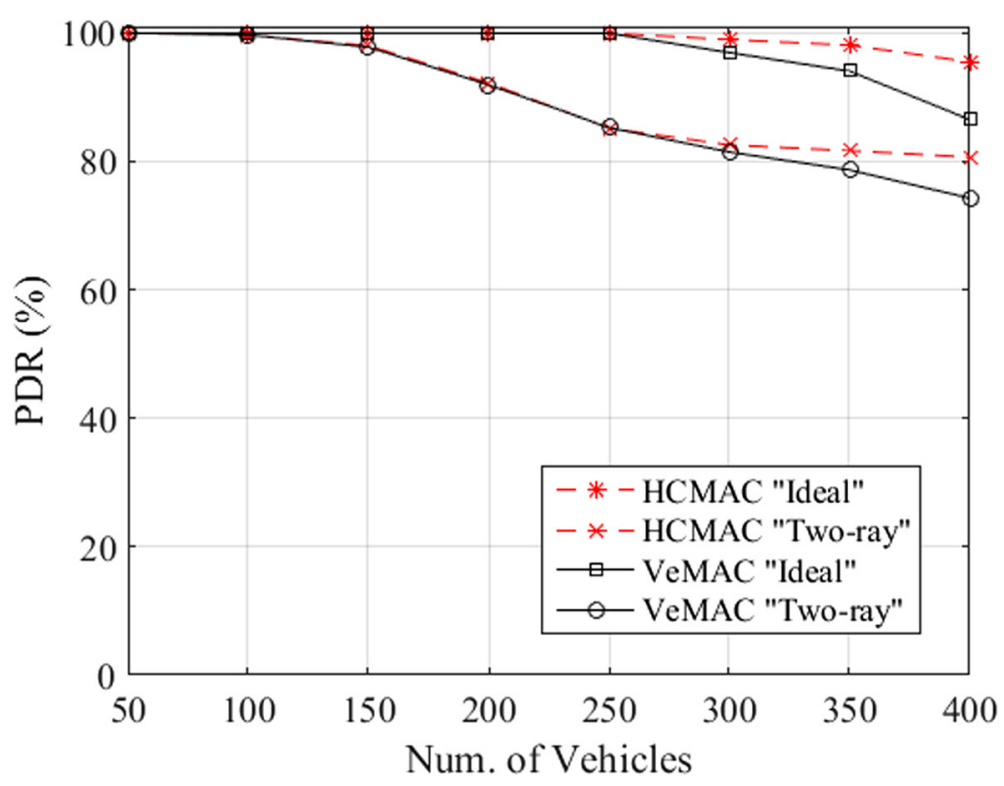

Fig. 22 PDR "highway scenario"

For example, for the ideal channel case, when the number of vehicles is 400 , the average transmission interval of HCMAC is around $135 \mathrm{~ms}$ compared to $155 \mathrm{~ms}$ for VeMAC. This is attributed to the observation that VeMAC experiences more collisions while it takes more frames to detect the collisions than HCMAC. This makes the transmitter repeatedly select occupied slots leading to a longer transmission interval.

Figure 23 also demonstrates the maximum transmission interval which indicates that HCMAC's capability to reduce the transmission interval also keeps its maximum transmission interval of each vehicle bounded to much smaller value compared to VeMAC. For example, for the ideal channel case, when the number of vehicles is 400 , the maximum transmission interval is around $900 \mathrm{~ms}$ for HCMAC compared to $1300 \mathrm{~ms}$ for VeMAC.

For the two-ray model, each protocol experiences a very small decrement in the average and maximum transmission intervals compared to the ideal channel case due to the small decrement in the number of

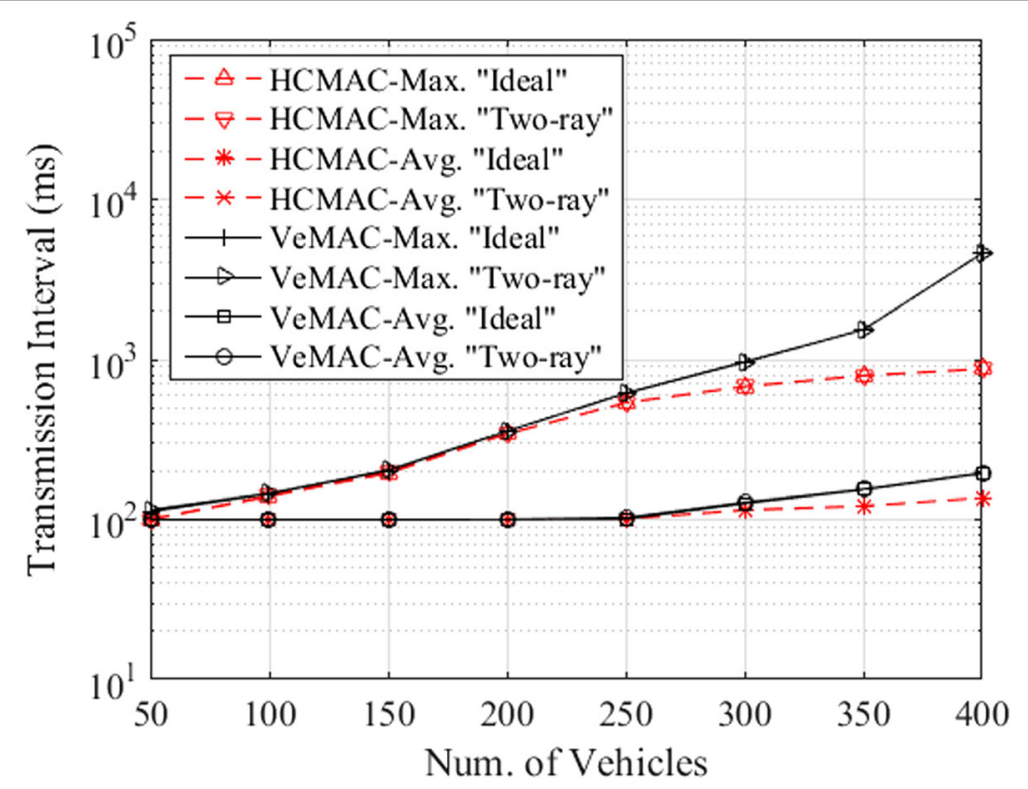

Fig. 23 Transmission interval "highway scenario" 
collision events as shown in Fig. 20 (with the log scale, Fig. 23 shows completely overlapping plots).

\subsubsection{Simulation results under urban scenarios}

We evaluated the network performance under the urban scenarios in a way similar to the highway scenarios. Both HCMAC and VeMAC are evaluated over a wide range of vehicle densities.

Figure 24 shows the average number of collision events experienced by all the vehicles within a single TDMA frame for both channel models: ideal and two-ray ground models. As the network becomes denser with the increased number of vehicles, HCMAC's collision detection technique becomes more effective. In Fig. 24, for the ideal channel case, when the number of vehicles is 650 , HCMAC has on average 3 collisions per frame, while VeMAC incurs as many as 17 collisions per frame. Similar to the highway scenario, for the two-ray channel case, each protocol has fewer collisions compared to the ideal channel case, due to the same packet capture effect that has been explained in the highway scenario.

HCMAC's capability of collision reduction also enhances the performance metrics: the throughput, the PDR, and the transmission intervals. Figure 25 compares the throughput. For the ideal channel case, when the vehicle density is 650, HCMAC achieves a throughput of around 69 successful transmissions per frame compared to a throughput of 54 for VeMAC. Figure 25 also shows that with employing the two-ray channel model, when the number of vehicles is 650, HCMAC has a throughput of around 54 packets per frame compared to a throughput of 50 for VeMAC. Figure 26 analyzes the PDR performance. As the number of vehicles increases from 250 to 650, the PDR dramatically decreases in the VeMAC case (i.e., the PDR decreases from 97 to $65 \%$ over the ideal channel and from 97 to $60 \%$ over the two-ray channel). In the HCMAC case, in contrast, for the same range of density, the PDR decreases only from 99 to $91 \%$ over the ideal channel and from 98 to $80 \%$ over the two-ray.

Similar to the highway scenario, for both channel models, each protocol has almost the same performance of transmission interval.

Figure 27 illustrates the average transmission interval versus the vehicle density. In the dense networks, HCMAC achieves lower transmission interval than VeMAC.

For example, when the number of vehicles is 650 , the average transmission interval is around $150 \mathrm{~ms}$ compared to $210 \mathrm{~ms}$ for VeMAC. The maximum transmission interval is also illustrated in Fig. 27, which demonstrates that HCMAC can substantially reduce the maximum transmission intervals for all individual vehicles. For example, when the number of vehicles is 650, the maximum transmission interval for HCMAC is around $1180 \mathrm{~ms}$, a reduction of $77 \%$ compared with $3578 \mathrm{~ms}$ for VeMAC.

\section{Conclusion and future work}

In this paper, we presented a Hybrid Cooperative MAC (HCMAC) protocol for vehicular networks. HCMAC is aimed at providing a reliable broadcast service for dense vehicle-to-vehicle networks, which is regarded as highly

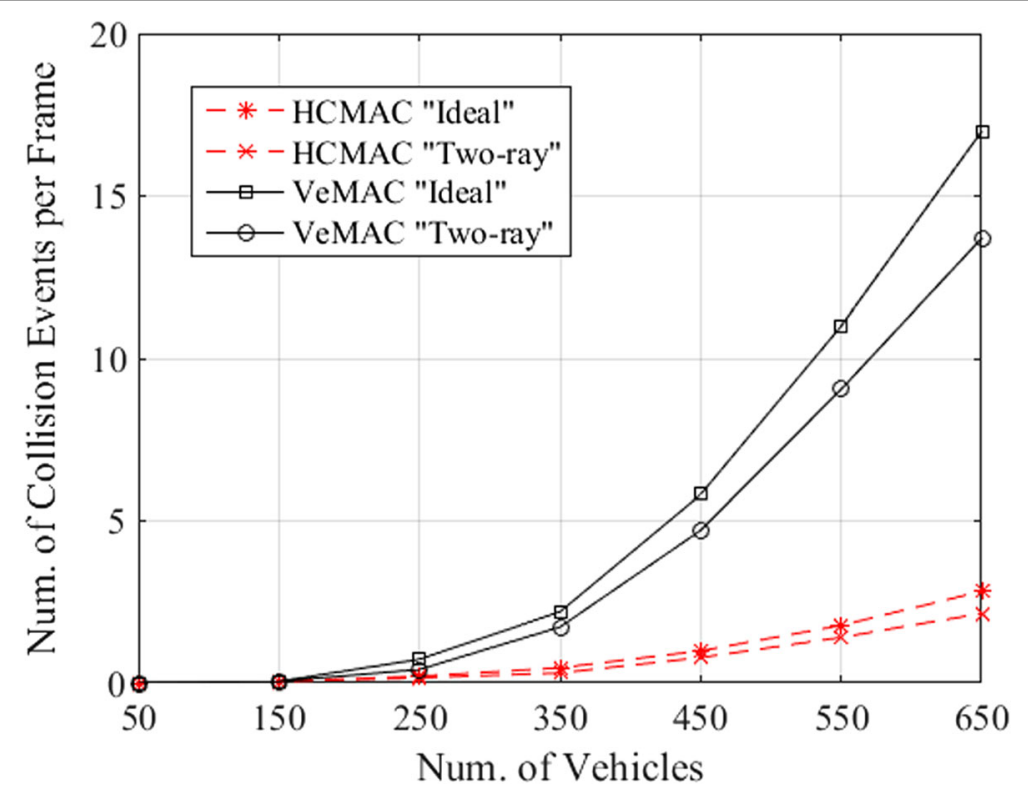

Fig. 24 Number of collision events "urban scenario" 


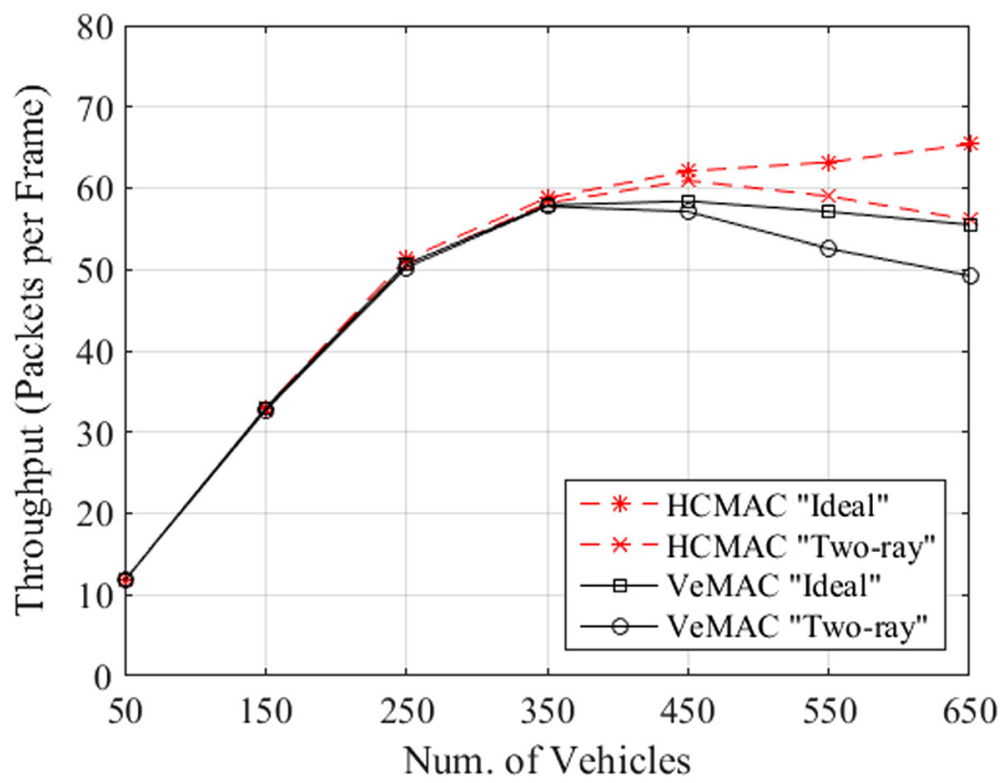

Fig. 25 Throughput "urban scenario"

challenging but is increasingly demanded for the vehicle safety applications. HCMAC integrates a TDMA protocol with a CSMA's collision avoidance strategy to provide highly efficient channel access with a substantially reduced collision. In addition, we introduced new collision detection mechanisms based on status feedback of neighbors and time slots, which further reduce the probability of collisions. We analyzed the time slot acquisition process and derived an analytic model for the collision probability of the proposed HCMAC protocol, whose accuracy is verified against network simulations. We evaluated the performance of HCMAC in comparison with VeMAC, a well-known TDMA protocol. The presented analytic model shows that HCMAC achieves substantially higher packet delivery ratio (PDR) and lower collision rate than VeMAC under various network conditions. In addition, we implemented both HCMAC and VeMAC in a realistic network simulator and conducted an extensive set of simulations with a wider range of network examples under highway and urban

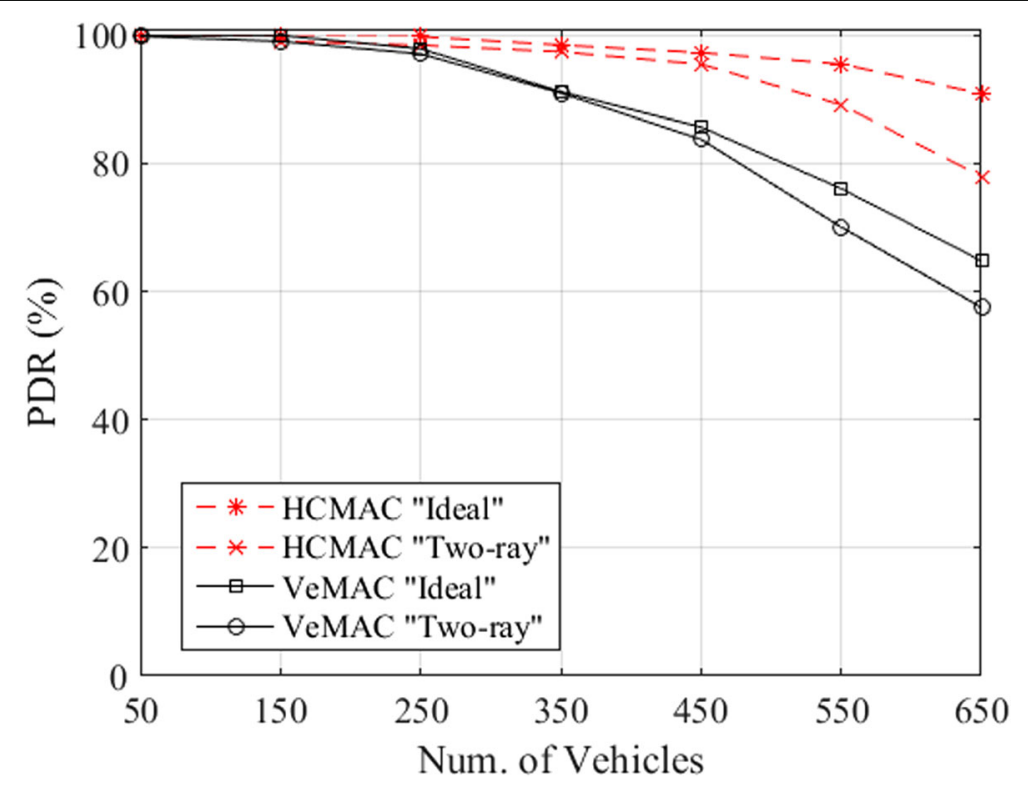

Fig. 26 PDR "urban scenario" 


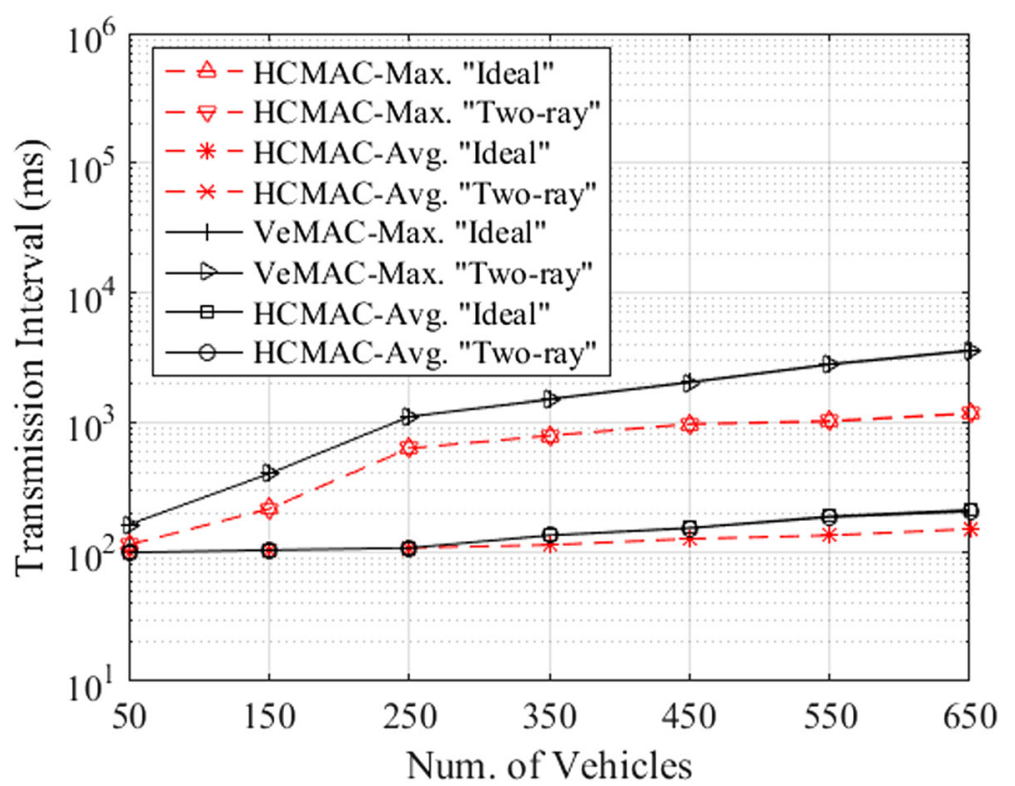

Fig. 27 Transmission interval "urban scenario"

scenarios. The simulation results demonstrated that HCMAC outperforms VeMAC in all aspect of metrics evaluated, which include collision rate, throughput, PDR, and transmission intervals.

In the future, we plan to develop a dynamic scheduling process that can determine the optimal slot number, and wireless range along with the network density changes utilizing the proposed analytic model.

\section{Endnotes}

${ }^{1}$ For a data rate of $12 \mathrm{Mbps}$, DSRC standard defines an SNR threshold of $15 \mathrm{~dB}$ [28].

${ }^{2}$ Videos of our simulator have been recorded and uploaded to $[29,30]$.

\section{Abbreviations}

AIS: Automatic Identification System; BSM: Basic Safety Message; CCH: Control Channel; CDMA: Code Division Multiple Access; CS: Carrier Sense; CSMA: Carrier Sense Multiple Access; CTS: Clear To Send; CW: Contention Window; DOT: U.S. Department of Transportation; DS: Detected Slots; DSRC: Dedicated Short-Range Communications; EDCA: Enhanced Distributed Channel Access; FCC: Federal Communication Commission; FCW: Forward Collision Warning; GPS: Global Position System; HCMAC: Hybrid Cooperative MAC; IMA: Intersection Management Assist; ITS: Intelligent Transportation System; LCW: Lane Change Warning; LTA: Left Turn Assist; NHTSA: National Highway Traffic Safety Administration; OBU: Onboard Unit; PDR: Packet Delivery Ratio; PN: Pseudo Noise; RSSI: Received Signal Strength Indicator; RSU: Road Side Unit; RTS: Request To Send; SAE: Society of Automotive Engineers; SCH: Service Channel; TDMA: Time Division Multiple Access; V2l: Vehicle-to-infrastructure; V2V: Vehicle-to-vehicle; VANET: Vehicular ad hoc network

\section{Acknowledgements}

This research was supported by the Center for Integrated Smart Sensors funded by the Ministry of Science, ICT\& Future Planning as Global Frontier Project (CISS-2018), and it was also supported by Institute for Information \& communications Technology Promotion (IITP) grant funded by the Korea government (MSIT) with grant number [R7117-16-0164], Development of wide area driving environment awareness and cooperative driving technology which are based on V2X wireless communication.

\section{Funding}

Not applicable.

\section{Availability of data and materials} Not applicable.

\section{Authors' contributions}

MAAEG is the primary author of this work. MAAEG and ME contributed to the conception of the study and performed the system analysis. MAAEG developed the simulator environment and conducted all the simulations. MAAEG wrote the manuscript. HWK provided great comments to enhance the paper quality. All authors reviewed and approved the final manuscript.

\section{Competing interests}

The authors declare that they have no competing interests.

\section{Publisher's Note}

Springer Nature remains neutral with regard to jurisdictional claims in published maps and institutional affiliations.

\section{Author details}

${ }^{1}$ Department of Electronics Engineering, Chungbuk National University, Cheongju, South Korea. ${ }^{2}$ National Telecommunication Institute, Cairo, Egypt. ${ }^{3}$ Electrical Engineering Department, Al-Azhar University, Cairo, Egypt.

Received: 12 April 2018 Accepted: 21 February 2019

Published online: 15 March 2019

\section{References}

1. The World Health Organization, "The World Health report 2015 - reducing risks, promoting healthy life." Available online: http://www.who.int/whr/ 2002/chapter4/en/index7.html. Accessed 30 June 2017.

2. U.S. Department of Transportation, Bureau of Transportation Statistics, Available online: https:/www.rita.dot.gov/bts/sites/rita.dot.gov.bts/ files/ publications/national_transportation_statistics. Accessed 10 Nov 2017.

3. The CAMP Vehicle Safety Communications Consortium, "vehicle safety communications project task 3 final report," CAMP, Farmington Hills, MI, USA, Tech. Rep. DOT HS 809 859, Mar. 2005. 
4. IEEE Standard for Information technology-- local and metropolitan area networks-- specific requirements-- part 11: wireless LAN Medium Access Control (MAC) and physical layer (PHY) specifications amendment 6: wireless access in vehicular environments, IEEE, pp.1-51, July 2010.

5. IEEE Standard for Wireless Access in Vehicular Environments (WAVE) -networking services, in IEEE Std 1609.3-2016 (Revision of IEEE Std 1609.32010), IEEE, pp.1-160, April 2016.

6. IEEE Standard for Wireless Access in Vehicular Environments (WAVE) -multi-channel operation, in IEEE Std 1609.4-2016 (Revision of IEEE Std 1609. 4-2010), IEEE, pp.1-94, March 2016.

7. Dedicated Short Range Communications (DSRC) Message Set Dictionary, Available online: http//standards.sae.org/22735_201603/. Accessed 20 Oct 2017.

8. S. Eichler, Performance Evaluation of the IEEE 802.11p WAVE Communication Standard, Proc. IEEE 66th Vehicular Technology Conf. (VTC 'O7-Fall), pp. 2199-2203, 2007.

9. F. Borgonovo, A. Capone, M. Cesana and L. Fratta, RR-ALOHA, a reliable RALOHA broadcast channel for ad-hoc inter-vehicle communication networks, In Proceedings of Med-Hoc-Net, 2002.

10. F. Borgonovo, A. Capone, M. Cesana, L. Fratta, ADHOC MAC: new MAC architecture for ad hoc networks providing efficient and reliable point-topoint and broadcast services. Wirel. Netw 10, 359-366 (July 2004).

11. H.A. Omar, W. Zhuang, L. Li, VeMAC: a TDMA-based MAC protocol for reliable broadcast in VANETs. IEEE Trans. Mob. Comput. 12(9), 1724-1736 (2013).

12. L. Gallo, and H. Jérôme, Analytical study of self organizing TDMA for V2X communications. In Communication Workshop (ICCW), IEEE International Conference on, pp. 2406-2411, 2015.

13. K. Bilstrup, E. Uhlemann, E.G. Ström, U. Bilstrup, On the ability of the $802.11 \mathrm{p}$ MAC method and STDMA to support real-time vehicle-to-vehicle communication. EURASIP J. Wireless Commun. Netw. 2009, 1-13 (2009).

14. Technical characteristics for universal shipborne automatic identification system using time division multiple access in the VHF maritime mobile band, Std. Recommendations ITU-R M.1371-1, 2006.

15. S. Bharati, W. Zhuang, CAH-MAC: cooperative adhoc MAC for vehicular networks. IEEE J. Sel. Areas Commun. 31(9), 470-479 (2013).

16. J. Huang, Q. Li, S. Zhong, L. Liu, P. Zhong, J. Wang, J. Ye, Synthesizing existing CSMA and TDMA based MAC protocols for VANETs. Sensors 17, 338-355 (2017).

17. M. Hadded, P. Muhlethaler, A. Laouiti, R. Zagrouba, L.A. Saidane, TDMAbased MAC protocols for vehicular ad hoc networks: a survey, qualitative analysis, and open research issues. IEEE Commun Surv Tutorials 17(4), 24612492 (2015).

18. X. Fan, C. Wang, J. Yu, K. Xing, Y. Chen, J. Liang, A reliable broadcast protocol in vehicular ad hoc networks. Int J Distrib Sens Netw 2015(8), 1-14 (2015).

19. J.J. Blum, A. Eskandarian, A reliable link-layer protocol for robust and scalable intervehicle communications. IEEE Trans. Intell Transp Syst 8(1), 4-13 (2007).

20. F. Watanabe, M. Fujii, M. Itami, K. Itoh, An analysis of incident information transmission performance using MCS/CDMA scheme. Proc. IEEE Intelligent Vehicles Symp. (IV '05), 249-254 (2005).

21. H. Nakata, T. Inoue, M. Itami, and K. Itoh, A study of inter vehicle communication scheme allocating PN codes to the location on the road, Proc. IEEE Intelligent Transportation Systems Conf. (ITSC '03), vol. 2, pp. 1527-1532, 2003.

22. J. B. Kenney, Dedicated short-range communications (DSRC) standards in the United States, Proceedings of the IEEE, vol. 99, no. 7, pp. 1162-1182, 2011.

23. K.F. Hasan, Y. Feng, Y. Tian, GNSS time synchronization in vehicular ad-hoc networks: benefits and feasibility. IEEE Trans. Intell. Transp. Syst.. https://doi. org/10.1109/TTS.2017.2789291.

24. M.A. Abd El-Gawad, and H. W. Kim, Reliable broadcast protocol based on scheduled acknowledgments for wireless sensor networks, The Institute of Electronics and Information Engineers (IEIE) Conference, pp. 485-489, 2017.

25. M. Elsharief, M.A. Abd El-Gawad, H.W. Kim, Density table based synchronization for multi-hop wireless sensor networks. IEEE Access 6 , 1940-1953 (2018).

26. C. Campolo, A. Vinel, A. Molinaro, Y. Koucheryavy, Modeling broadcasting in IEEE 802.11 p/WAVE vehicular networks. IEEE Commun Lett 15(2), 199-201 (2011).

27. H.A. Omar, W. Zhuang, L. Li, VeMAC: a novel multichannel MAC protocol for vehicular ad hoc networks. Proc. IEEE INFOCOM, 413-418 (2011).

28. H. Zhou et al., Toward multi-radio vehicular data piping for dynamic DSRC/ TWWS spectrum sharing. IEEE J Sel Areas Commun 34, 2575-2588 (2016).

29. [Online]. Available: https://youtu.be/JT14WJegGSk. Accessed 5 Mar 2019 .

30. [Online]. Available: https://youtu.be/PlegaReO-c8. Accessed 5 Mar 2019.

\section{Submit your manuscript to a SpringerOpen ${ }^{\circ}$ journal and benefit from:}

- Convenient online submission

- Rigorous peer review

- Open access: articles freely available online

- High visibility within the field

- Retaining the copyright to your article

Submit your next manuscript at $\boldsymbol{\nabla}$ springeropen.com 